\title{
Imperfection sensitivity of column instability revisited
}

\author{
Ana M. Girão Coelho ${ }^{\mathrm{a}, \mathrm{b},{ }^{*}}$, Pedro D. Simão ${ }^{\mathrm{a}, \mathrm{c}}$ and M. Ahmer Wadee ${ }^{\mathrm{d}}$ \\ ${ }^{a}$ Institute of Computers and Systems Engineering of Coimbra (INESC-Coimbra), Rua Antero de Quental 199, 3000-033 \\ Coimbra, Portugal (a.m.girao@clix.pt and pedro@dec.uc.pt) \\ ${ }^{\mathrm{b}}$ Department of Structural and Building Engineering - Steel and Timber Structures, Faculty of Civil Engineering and \\ Geosciences, Delft University of Technology, PO Box 5048, 2600 GS Delft, The Netherlands \\ ${ }^{\mathrm{c}}$ Department of Civil Engineering, University of Coimbra, Rua Luís Reis Santos, 3030-788 Coimbra, Portugal \\ ${ }^{\mathrm{d}}$ Department of Civil and Environmental Engineering, Imperial College London, London SW7 2AZ, United Kingdom \\ (a.wadee@imperial.ac.uk) \\ * Corresponding author at: Institute of Computers and Systems Engineering of Coimbra (INESC-Coimbra) \\ Rua Antero de Quental 199, 3000-033 Coimbra, Portugal \\ Phone: +351239790312/+351964837943; Fax: +351239790311 \\ Email: a.m.girao@clix.pt
}

\begin{abstract}
The buckling of columns is the classic problem in structural stability. It has been studied by many researchers over a large number of years, and it is well known that the severity of the buckling response can be greatly amplified by initial geometric imperfections in the column shape. The current paper presents and discusses the effects of imperfection shape, orientation and magnitude on the buckling behaviour of columns. Analyses are conducted for elastic columns with overall initial imperfections in the form of out-of-straightness and sway displacements, as well as local imperfections that, for instance, model constructional and material defects. Traditionally, the initial imperfections are modelled with the first buckling mode with a size selected according to fabrication tolerances. This approach will not necessarily provide a lower limit to the column prebuckling stiffness and strength. These assertions are supported by numerical results for imperfection-sensitive columns. The influence of end restraint on column strength is also studied since columns in actual frameworks are connected to other structural members such that their ends are restrained.
\end{abstract}

Keywords: Column buckling; Imperfection sensitivity; Localized defects; Boundary conditions

\section{Introduction}

The pioneering investigation by Euler on the elastic stability of a mathematically straight, prismatic, pin-ended, concentrically loaded, slender column founded the development of the "classical" theory of elastic buckling, in which the governing equations of the problem are linear and homogeneous in the lateral displacement $w$ and its first three derivatives, leading typically to a eigen-boundary-value 
problem [1]. The critical buckling loads are the eigenvalues and the corresponding buckling mode shapes are the eigenvectors of the problem. Practical columns, however, deviate from this ideal model due to the inevitable presence of geometrical, material, structural and load related imperfections that act to reduce the column load-carrying capacity. For modelling purposes, geometrical, material and structural imperfections are usually considered by means of an equivalent initial geometric imperfection, in the form of an initial curvature or out-of-straightness (lateral deflection of the column relative to the undeformed state) and an initial sway imperfection (relative lateral displacement between the column ends) [2-4].

The buckling load of a compressed ideal column is also affected by the boundary conditions. For all possible boundary conditions, the critical load can be always related to the basic pin-ended column element through the concept of the effective length, $L_{\text {eff }}$ that was first introduced by Jasinsky in 1893 [5]. The effective length is defined as the length of a pin-ended column that has the same critical load as a column with other prescribed end-conditions. This concept allows codes of practice to be simplified considerably - see, for instance, the American specification for structural steel buildings [6] and the European code of practice for the design of steel structures, EN 1993 [7], with rules only necessary for pin-ended columns. In fact, much of the research on elastic buckling of columns is based on the behaviour of this simple column. In particular, and among the many analyses of imperfection sensitive columns, the majority is concerned with the buckling of a simple uniform column with equivalent initial geometric imperfections and eccentric axial loads. Most studies consider an initial centreline deflection in the form of a half-sine curve, $\delta_{0} \sin \left(\pi x / L_{\text {eff }}\right)$, where $\delta_{0}$ is the initial out-of-straightness at the middle of the pin-ended equivalent column. This is a simplified form of geometric imperfections that uses a single buckling mode representation of the imperfection in which the amplitude $\delta_{0}$ is allowed to vary according to the fabrication tolerances stipulated in the applicable engineering standards [8], or in some cases being treated as a random variable [9-10].

The equivalent geometric imperfections change the column response. An imperfect column exhibits a limit point with bending being introduced from the onset of loading. Since the bending effect is likely to be secondary compared to the effect of the axial force, it is current practice to assume that the characteristic column strength is reached when the material yield stress $f_{\mathrm{y}}$ is first attained at any point in the column; in the context of the Ayrton-Perry formulation [11], the maximum stress $\sigma_{\max }$ is given by:

$$
\sigma_{\max }=\frac{N_{\mathrm{Ed}}}{A}+\frac{M_{\text {max }}}{W_{\mathrm{el}}} \leq f_{\mathrm{y}}
$$

where $N_{\mathrm{Ed}}$ is the applied axial compressive load, $A$ is the area of cross section, $W_{\mathrm{el}}=A i^{2} / c$ is the section modulus corresponding to the fibre with maximum elastic stress with $i$ being the section radius of gyration and $c$ being the distance from neutral axis to extreme fibre, and $M_{\max }$ is the maximum bending moment in the column. The theory that effectively combines the Ayrton-Perry approach to failure of columns with a global imperfection parameter, as proposed by Robertson [12] for the definition of the buckling resistance of columns, is essentially elastic in nature [11] and leads to the well-known column curves based on the effective length concept that are adopted in 
modern design codes. The EN 1993 document, in particular, adopts a single equation for the column design curves developed by Rondal and Maquoi [13]; these describe the practical compressive strength of a column in terms of the slenderness ratio $\bar{\lambda}=\sqrt{N_{\mathrm{pl}} / N_{\mathrm{cr}}}$, where $N_{\mathrm{cr}}$ is the elastic critical load and $N_{\mathrm{pl}}$ is the squash load.

The important problem of determining consistent equivalent geometric imperfections includes the choice of their shape and size and its relation to the column end restraints. The equivalent initial imperfections are usually assumed to be proportional to the classical buckling mode (or as a linear combination of the relevant critical modes), but in fact these are functions involving uncertainties that may have a random nature. In principle, the choice of the imperfection shape that leads to the lowest column strength subjected to amplitude constraints should involve a Fourier-type approach, which usually gives a good interpretation of the actual column imperfections [9].

With this range of issues in mind, the research presented currently has the following specific objectives:

1. To dissociate the form of initial geometric imperfections from the traditional approach of assuming the imperfection shape affine to the lowest bifurcation mode while bounded by a given imperfection amplitude.

2. To include the effect of a localized deflection pattern within the problem domain (i.e. a localized geometric imperfection to a section of the column). Such localized effects may arise in steel columns, for example, due to welding of a reinforcing steel plate over a short column length, which would modify the residual stress distribution, for example. This effect is then combined with the general distributed imperfection pattern along the column length to compare the strength ratios to the critical load.

3. To examine the influence of end restraint on the column strength and behaviour. More specifically, two aspects of restrained column behaviour are considered: the magnitudes of the rotational and sway end stiffnesses, plus the form and bow amplitude of the initial geometric imperfections.

4. To compare the effects of the various imperfections and end support conditions by using the maximum elastic strength as the relative measure and then derive design sensitivities of the critical load factor for the various studies. A parametric study is conducted through computational analysis and results are then established.

As far as the authors are aware, little attention has been paid to this class of problems, and the physical results are in themselves of interest. In particular, it is shown that for a given imperfection amplitude, the shape of such an imperfection is an important factor in the column strength ratios to the buckling load. These effects are examined with exact treatments through extensive computational modelling.

The buckling formulation used, as well as the underlying computational implementation, can be easily extended to other types of structures. For example: thin-plated structures, columns on elastic foundations, sandwich struts and prestressed stayed columns; the sensitivity to the geometry of the imperfection of the latter three components have been investigated to some extent already [14-16]. 


\section{Elastic buckling of uniform columns}

The elastic buckling of a perfect column is a classic textbook problem, see [2-3,17]. Columns that are very slender do not have a significant sensitivity to initial geometric imperfections; only when yielding comes into the picture does the sensitivity show itself. This is significant for moderately slender columns. In that range of slenderness, an imperfect column has a load-carrying capacity that is usually significantly less than that obtained from calculating the buckling load of a column with a perfect geometry.

\subsection{Basic equations}

Consider an elastically supported column with initial deformations associated with out-ofstraightness of the column, $w_{\delta 0}(x)$, and initial sway imperfection, $\Delta_{0}$ (see Fig. 1). The following assumptions are made:

1. The analysis is purely elastic with the stress-strain relationship being completely linear and $E$ is defined as the Young modulus.

2. The column is of uniform cross-section with $I$ being the second moment of area.

3. The column is stress-free in its initial configuration, before the application of the axial load.

4. The column is considered transversely supported so that the possibility of buckling about the weak axis is precluded.

5. Local instabilities do not occur.

6. Shear deformations are not accounted for.

7. The curvature is approximated by $\frac{d^{2} w}{d x^{2}}$ because the rotations of the member are small so that the term $\left(\frac{d w}{d x}\right)^{2}$ is negligible compared to unity in the expression for the actual curvature,

$$
\frac{\frac{d^{2} w}{d x^{2}}}{\left[1+\left(\frac{d w}{d x}\right)^{2}\right]^{\frac{3}{2}}} \text {. }
$$

The column is loaded axially by a compressive load $N_{\text {Ed }}$ that retains its direction as the column deflects. The total deflection $w_{\mathrm{T}}(x)$ is obtained by superimposing the lateral deflection $w(x)$ to the initial geometric imperfections:

$$
w_{\mathrm{T}}(x)=\underbrace{w_{\delta 0}(x)+w_{\Delta 0}(x)}_{w_{0}(x)}+w(x) .
$$

The column ends a and $\mathrm{b}$ are restrained by rotational and longitudinal springs, with $K_{\theta}$ being the corresponding rotational spring constant and $K_{\Delta}$ being the longitudinal spring. For the numerical studies presented later, the stiffnesses $K_{\theta}$ and $K_{\Delta}$ are specified in a non-dimensional form $\left(k_{\theta}\right.$ and $\left.k_{\Delta}\right)$ in terms of $\frac{E I}{L}$ and $\frac{E I}{L^{3}}$ of the column, respectively. 
The mathematical formulation of this problem is now presented. Figure 2 shows the free-body diagram of an infinitesimal segment of this column. The moment equilibrium equation is written in the following form [4]

$$
\frac{d^{2} M}{d x^{2}}-N_{\mathrm{Ed}} \frac{d^{2}\left(w_{\mathrm{T}}\right)}{d x^{2}}=\frac{d^{2} M}{d x^{2}}-N_{\mathrm{Ed}} \frac{d^{2}\left(w+w_{\delta 0}+\frac{\Delta_{0}}{L} x\right)}{d x^{2}}=0 .
$$

From Euler-Bernoulli bending theory the moment-curvature relationship is

$$
M=-E I \frac{d^{2} w}{d x^{2}}
$$

From Eqs. (3) and (4) we obtain the general fourth-order differential equilibrium equation, which is applicable to columns with any boundary conditions,

$$
\frac{d^{2}}{d x^{2}}\left(-E I \frac{d^{2} w}{d x^{2}}\right)-N_{\mathrm{Ed}} \frac{d^{2} w}{d x^{2}}=N_{\mathrm{Ed}} \frac{d^{2} w_{0}}{d x^{2}} \quad \text { or } \quad w^{\mathrm{IV}}+\mu^{2} w^{\prime \prime}=-\mu^{2} w_{0}^{\prime \prime}
$$

where $\mu$ is given by

$$
\mu^{2}=\frac{N_{\mathrm{Ed}}}{E I}
$$

\subsection{Form of initial geometric imperfections}

It is well known that perfect columns are just idealized models. Typically, they have an imperfect geometry and the applied loads do not pass through the centroid of the column cross section precisely. In this section, generic column imperfections in the form of equivalent initial geometric imperfections are considered.

In practice, it is common to consider geometric imperfection shapes affine with the lowest bifurcation mode or as a linear combination of the $n_{\mathrm{cr}}$ lowest bifurcation modes,

$$
w_{\delta 0}(x)=\sum_{i=1}^{n_{\mathrm{cr}}} \delta_{0, i} w_{\mathrm{cr}, i}(x)
$$

whereby $\delta_{0, i}$ and $w_{i}(\mathrm{x})$ are the amplitude and the shape of the $i^{\text {th }}$ mode, defined as follows

$$
w_{\mathrm{cr}, i}(x)=a_{\mathrm{cr}, i} \sin \left(\mu_{\mathrm{cr}, i} x\right)+b_{\mathrm{cr}, i} \cos \left(\mu_{\mathrm{cr}, i} x\right)+c_{\mathrm{cr}, i} x+d_{\mathrm{cr}, i}
$$

and

$$
\mu_{\mathrm{cr}, i}=\sqrt{\frac{N_{\mathrm{cr}, i}}{E I}}
$$

where $N_{\mathrm{cr}, i}$ is the $i^{\text {th }}$ critical load of the system and $w_{\mathrm{cr}, i}$ is the general solution of the governing differential equation for the perfect column system. This strategy has no actual physical or mathematical reasoning. The underlying philosophy of this approach has its basis in a mathematical simplification for finding the particular solution of the governing differential equation. The particular solution will not necessarily satisfy the column boundary conditions; however, if a general solution is found to the homogeneous form of the same equation, then it can be used to satisfy the boundary conditions.

Equivalent geometric imperfections are intrinsic to the member itself and are independent of the 
column boundary conditions. We can then anticipate that the load-deflection response of an imperfect column will strongly depend on the boundary conditions, in the sense that for some boundary condition types the degradation of the column strength for a specific imperfection shape can be more severe than for others. Additionally, since the initial shape of the equivalent geometrical imperfection is random in form and magnitude, the buckling modes do not necessarily constitute a complete set of functions that describe a general curve along the column length. Since the initial shape is continuous and has a finite number of extrema in the range $0<x<L$, then the initial bow is best represented by a Fourier sine series where there is no initial displacement at the boundaries [18],

$$
w_{\delta 0}(x)=\sum_{n=1}^{\infty} \delta_{n} \sin \frac{n \pi x}{L}
$$

and thus

$$
w_{0}(x)=\frac{\Delta_{0}}{L} x+\sum_{n=1}^{\infty} \delta_{n} \sin \frac{n \pi x}{L} .
$$

\subsection{Solution of the equilibrium equation}

As indicated in the previous section, the complementary solution of the differential equilibrium equation for the perfect system is

$$
w_{\mathrm{c}}(x)=a \sin (\mu x)+b \cos (\mu x)+c x+d
$$

where $a, b, c$ and $d$ are integration constants to be determined using the following boundary conditions (Fig. 1)

$$
\begin{array}{ll}
\text { at } x=0 & w=0 \\
& -E I w^{\prime \prime}=-K_{\theta \mathrm{a}} w^{\prime} \\
\text { at } x=L & -E I w^{\prime \prime \prime}-N_{\mathrm{Ed}}\left(w^{\prime}+w_{0}^{\prime}\right)=-K_{\Delta \mathrm{b}} w \\
& -E I w^{\prime \prime}=K_{\theta \mathrm{b}} w^{\prime}
\end{array}
$$

with the particular integral $w_{\mathrm{p}}$ for this equation being obtained by using the method of undetermined coefficients. This leads to

$$
w_{\mathrm{p}}(x)=\sum_{n=1}^{m} \frac{N_{\mathrm{Ed}}}{N_{\mathrm{cr}, i}-N_{\mathrm{Ed}}} \delta_{0, i} w_{\mathrm{cr}, i}(x)
$$

or

$$
w_{\mathrm{p}}(x)=\sum_{n=1}^{m} \frac{N_{\mathrm{Ed}}}{n^{2} \frac{\pi^{2} E I}{L^{2}}-N_{\mathrm{Ed}}} \delta_{n} \sin \frac{n \pi x}{L}
$$

if the initial bow imperfection is given by Eq. (7) or (10), respectively.

\subsection{Localized imperfections}

The influence of imperfections on column buckling is now analysed at the level of localized 
imperfections that are modelled in the form of a single wave soliton given by

$$
w_{0, \text { sol }}(x)=\delta_{0, \text { sol }} \operatorname{sech}^{2}\left(\varphi_{\text {sol }} \frac{x-\xi}{L}\right)
$$

where $\delta_{0 \text {,sol }}$ is the maximum wave amplitude, $\varphi_{\text {sol }}$ is a form parameter that makes the soliton narrower or wider, and $\xi$ is the abscissa of the soliton peak. A typical single wave soliton is shown in Fig. 3. This localized imperfection can be added to Eq. (11) to obtain a general form of the initial geometrically equivalent imperfection.

The solution of the equilibrium Eq. (5) now has to include the additional particular solution associated with the above localized wave. Since the differential operator related to Eq. (5) is linear, the particular solution of the equilibrium equation considering the generalized imperfection given by Eqs. (11) and (16) is the sum of the particular solutions associated with each term. Application of the method known as the variation of parameters [19], in the context of the symbolic algebraic manipulator Mathematica [20], and for $\varphi_{\text {sol }} \neq 0\left(\varphi_{\text {sol }}=0\right.$ is associated with a constant function, with no physical interest for this particular problem), the particular solution for the soliton term is given by

$$
w_{\mathrm{p}, \mathrm{sol}}(x)=i \frac{\delta_{\mathrm{sol}} L}{\varphi_{\mathrm{sol}}} \mu\left[{ }_{2} F_{1}\left(1,-i \frac{L \mu}{2 \varphi_{\mathrm{sol}}}, 1-i \frac{L \mu}{2 \varphi_{\mathrm{sol}}},-e^{2 \varphi_{\mathrm{sol}} \frac{x-\xi}{L}}\right)-{ }_{2} F_{1}\left(1, i \frac{L \mu}{2 \varphi_{\mathrm{sol}}}, 1+i \frac{L \mu}{2 \varphi_{\mathrm{sol}}},-e^{2 \varphi_{\mathrm{sol}} \frac{x-\xi}{L}}\right)\right]
$$

whereby ${ }_{2} F_{1}$ is an hypergeometric function [21] with complex variables but, together with its derivative of any order, $w_{\mathrm{p}, \text { sol }}$ shows real values only for any value of $x$. Having found the particular solution of the equilibrium equation, the eigen-boundary value problem that computes the equilibrium paths follows the same methodology as presented in Section 2.3.

\section{Imperfection sensitivity studies}

To understand the sensitivity of columns to geometric imperfections better and to characterize the critical conditions fully, it is important to investigate the effects of initial geometric imperfections of specified amplitude and shape.

The results of a parametric investigation are compared with those corresponding to a basic problem, which currently is taken as a HEB240 section buckling about its major axis and simply supported at the ends, with a length of $8 \mathrm{~m}(\bar{\lambda}=1.02)$. This example was specifically chosen since a slenderness parameter approximately equal to unity is the value that produces the greatest sensitivity in columns. The shape of the initial deflection is assumed to be that of a half sine-wave. The basic problem is summarized in Fig. 4.

A series of calculations is first performed using different values of initial central deflection. The basic problem assumes a value of $\delta_{0}$ of $L / 500$ that corresponds to the tolerance for geometrical imperfections in the European execution specification EN 1090 [8]. Three additional values are chosen, namely $L / 1000, L / 250$ and $L / 125$. Imperfections are introduced from eigenvalue analysis 
(imperfection patterns based on the first buckling mode shape) and Fourier sine series representations. The effect of localized imperfections is also taken into account.

The baseline conditions of end-restraint are modified as follows:

1. Columns without sway: fully fixed ends.

2. Columns with sway: fixed-free ends (cantilevered column) and fixed-transversely sliding ends.

\subsection{Imperfection shapes affine to the lowest bifurcation mode}

First, the column imperfection shape is assumed to be proportional to the first buckling mode. This is the simplest representation of the column geometric imperfection. The buckling behaviour of the column, including the deflected shape and the maximum load capacity, was quantified by the software Mathematica. Table 1 shows the calculated values of the ratio of the strength of the imperfect structure to that of the perfect structure $\left(N_{\text {max }} / N_{\text {cr }}\right)$.

The variation of the total central deformation of the pin-ended column with axial load is shown in non-dimensional form in Fig. $5 a$. The curves are expressed in terms of the normalized axial force in the column $\left(N_{\mathrm{Ed}} / N_{\mathrm{cr}}\right)$ versus the normalized maximum total lateral deflection of the member $\left(w_{\mathrm{T}, \max } / L\right)$. Curve 1 represents the basic case shown in Fig. 4 and is valid only over the region $\mathrm{AB}$, where the column behaves elastically, i.e. $\sigma_{\max } \leq f_{\mathrm{y}}-$ see Eq. (1). The remaining curves show the effect of larger and smaller initial amplitudes $\delta_{0}$. If $\delta_{0}$ is infinitesimally small, the behaviour of the column converges to that of the perfect column. Fig. $5 b$ shows that as the compressive axial load in the column is increased, the maximum stress defined in Eq. (1) increases at a progressively greater rate until, eventually, it reaches the yield stress (vertical line in the graph). If the column stress is increased further, a plastic zone forms on the concave side of the column and collapse ensues. The load at which $\sigma_{\max }=f_{\mathrm{y}}$ can be conservatively taken as the load capacity [22].

The buckling behaviour of columns for which the ends are not pinned is now examined in the context of the equivalent pin-ended column, by means of the effective length concept (see Fig. 6). The variation with the axial load of the ratio between the maximum total deflection of the equivalent pin-ended column and the effective length $L_{\text {eff }}$ is first analysed. As an example, the amplitude of the initial bow is taken as $L_{\text {eff }} / 500$. The graph in Fig. $7 a$ shows that the column behaviour is identical to that of a pin-ended column of length $L_{\text {eff }}$ irrespective of the boundary conditions. Now suppose that the axially loaded columns in Fig. 6 have an initial deformation $w_{\delta 0}$ that is affine to the first buckling mode, see Eq. (7), with $n_{\mathrm{cr}}=1$, which is now measured from the line joining the two ends. The characteristic features of the response $N_{\mathrm{Ed}} / N_{\mathrm{cr}}$ versus $w_{\mathrm{T} \text {,max }} / L$ are identical to the pin-ended column (Fig. 5) but the elastic limiting stress is clearly dependent on the end conditions and so the load-carrying capacity varies, as indicated in Figs. $7 b-d$.

\subsection{Imperfection representations by truncated Fourier sine series}

The column imperfection shapes are now modelled as Fourier sine series. The current work only 
considers initial imperfections in the form of a single half-sine wave $(n=1)$ and two half-sine waves $(n=2)$. These are shown in Fig. 8 for increasing bow amplitudes $\delta_{0}$ and for the particular case of a cantilevered column. Note that this specific example corresponds to a sway-permitted column and thus the load versus lateral relative displacement response is sensitive to the amount of initial sway displacement $\Delta_{0}$. That is not the case for sway-prevented columns as detailed in Appendix A.

\subsubsection{Fourier Sine series expansion with the series truncated at $n=1$}

Three different column configurations are studied (see Fig. 6). These cases cover the configurations for which the imperfection shape is not affine to the buckling mode. The parametric variations in sway imperfection and maximum initial bow amplitude are summarized in Table 2. The findings of this parametric study are summarized in Figs. 9 and 10 and Table 3. Some indication of the effect of varying $\delta_{0}$ and $\Delta_{0}$ is given in these figures where non-dimensional graphs of load versus maximum lateral deflection for the parameters set out in Table 2 are shown. As would be expected, the smaller the values of $\delta_{0}$ and $\Delta_{0}$, the higher the column load-carrying capacity, which is identified in each figure with enlarged circles. For all examples, the buckled configuration typically tends to the critical buckling mode, irrespective of the magnitude of the initial bow and sway imperfections. The results in terms of column strength ratios to the critical load are presented in Table 3.

For the fully fixed (sway-prevented) column, the maximum transverse deformation occurs at mid-height. The different bow amplitudes considered produced approximately parallel loaddeflection curves that approach the critical buckling case asymptotically for any given initial bow. However, the load-carrying capacity in these columns is severely reduced as the initial bow amplitude increases (Fig. 9a). The graph in Fig. $9 b$ shows that the maximum load in a swayprevented column is relatively insensitive to the initial sway displacement.

Fig. $10 a$ shows load-deflection curves for the cantilevered column (a sway-permitted column), expressed in terms of the normalized total deflection at the top of the column. The four lower curves in Fig. 10a(i) correspond to the example cases 7 to 10, see Table 2. These are referred to as the "negative" $\delta_{0}$ curves in the discussions that follow. Similarly, the other four curves (example cases 1 to 4 ) are associated with "positive" $\delta_{0}$. The "negative" $\delta_{0}$ curves are practically identical to the load-deflection curves represented in Fig. $7 b$, in which the imperfection shape is affine to the first buckling mode shape, although a significant reduction in the load-carrying capacity is observed. The second group of curves are plotted for "positive" $\delta_{0}$. These curves are particularly interesting: the amplitude $\delta_{0}$ has a favourable effect on the column load-carrying capacity and the column is deformed nearly in full reversed curvature bending under sidesway deflections, see also Fig. $10 a$ (ii). It can be seen that the relative orientation of the out-of-straightness and initial sway has a significant effect on the maximum strength for this column. The favourably oriented imperfection in the column from example 4 results in a strength ratio of 0.93 compared to 0.79 for the unfavourably orientated imperfection in the column from example 10, see Table 3.

The behaviour in Fig. 10b(i) is similar to that shown in the above figures with a notable exception: the load-deflection curves for a fixed-guided column are completely independent from 
the bow amplitude $\delta_{0}$. This happens because the magnitude of the initial bow $\delta_{0}$ has the strongest influence at the central zone of the column and not at the top where the maximum displacements occur. The load-carrying capacity however is greatly affected by this parameter and varies from 0.530 (for $\delta_{0}=-L / 125$ ) to 0.638 (for $\delta_{0}=L / 1000$ ) that represents a $17 \%$ variation. On the contrary and as expected, Fig. $10 \mathrm{~b}$ (ii) shows that fixed-guided columns are particularly sensitive to the initial sway imperfection.

\subsubsection{Fourier Sine series expansion with the series truncated at $n=2$}

Two basic column configurations are now analysed, corresponding to a pin-ended column and a fixed-guided column, that exhibit the same buckling load $\left(N_{\mathrm{cr}}=3456.5 \mathrm{kN}\right)$. The initial bow imperfection shape is given by Eq. (10) with two terms of the Fourier series,

$$
w_{\delta 0}(x)=\delta_{1} \sin \frac{\pi x}{L}+\delta_{2} \sin \frac{2 \pi x}{L}
$$

The Fourier coefficients $\delta_{1}$ and $\delta_{2}$ are found by assuming four different ratios, see Fig. 11. The parametric variations in sway imperfection and maximum initial bow amplitude are identical to those in Table 2. The comparison results are shown in Table 4.

The axial force-total deflection paths $\left(N_{\mathrm{Ed}} / N_{\mathrm{cr}}\right.$ versus $\left.w_{\mathrm{T}, \max } / L\right)$ obtained from the current analysis are shown in Figs. 12 and 13. These figures correspond to the pin-ended column $\left(w_{\mathrm{T}, \max }\right.$ occurs at mid-height of the column, $\left.w_{\mathrm{T}, 0.5 \mathrm{~L}}\right)$ and the fixed-guided column $\left(w_{\mathrm{T}, \max }\right.$ occurs at the guided top end of the column, $w_{\mathrm{T}, L}$ ), respectively. Initial bow amplitudes of $L / 500$ and $L / 125$ are assumed in graphs $a$ (i) and $a($ ii); initial sway displacements of $L / 200$ and $L / 500$ are considered in Figs. $a$ and $b$. In addition to the load paths, the column strength is shown in each figure with enlarged circles.

In both Figs. 12a, it can be observed that the buckling response is the exactly same for symmetric ratios $\delta_{1} / \delta_{2}$. This is a rather trivial remark since this column end restraint conditions are identical. It is significant however to note that as the initial bow amplitude increases, the column buckling behaviour becomes more sensitive to the imperfection shape, i.e. to the ratio $\delta_{1} / \delta_{2}$. These graphs also show that there is a loss of load-carrying capacity for increasing ratios $\delta_{1} / \delta_{2}$ and for the same value of $\delta_{0}$. This highlights the importance of the form of the initial imperfection, irrespective of the initial bow amplitude. The graph in Fig. $12 b$ again indicates that the strength in swayprevented columns is relatively insensitive to initial sway imperfections.

The curves in Fig. $13 a$ show identical results for a fixed-guided column. The response pattern is exactly the same as the one seen in Fig. 10b(i). However, the response is now sensitive to the ratios $\delta_{1} / \delta_{2}$ and so is the column load carrying capacity. It can be seen in graph $a(\mathrm{i})$ that the lower equilibrium curves correspond to the case where the maximum bow amplitude is closer to the transversely sliding end whereas in the upper curve the maximum initial bow amplitude is closer to the fixed end. When the imperfection becomes larger - graph $a$ (ii) - the buckling response changes dramatically. As for the cantilevered column in the previous section, the column is now deformed nearly in full reversed curvature bending. This happens as the ratio $\delta_{1} / \delta_{2}$ decreases. The reason for this may be related to the fact that a larger value of the out-of-straightness combined with a small $\delta_{1} / \delta_{2}$ ratio (see Fig. 11) allows the column deformation to converge to the first buckling mode 
easily. Finally, the diagram in Fig. $13 b$ confirms that this column is particularly sensitive to the initial sway magnitude.

\subsubsection{Comparisons}

It can be concluded from the previous results that for the range of parameters and conditions considered, the shape of geometric imperfections in the form of an initial bow had the most significant influence on the load capacity. The equilibrium paths in terms of axial force $N_{\mathrm{Ed}} / N_{\mathrm{cr}}$ versus relative deflection $w_{\max } / L$ are taken as a basis for comparing the results.

The key findings are summarized below:

1. Figs. $14 a$ (i) and (ii) show that pin-ended columns are relatively insensitive to the imperfection shape if no change in the initial curvature occurs. As can be seen in the graphs, the buckling responses and the column strength values with imperfection shapes affine to the first buckling mode almost coincide with those of the shapes represented by two term Fourier sine series that assume $\delta_{1} / \delta_{2}=4$, see Fig. 11 .

2. By including an initial out-of-straightness in the form of the first buckling mode shape with a magnitude of $L / 500$, a $30.2 \%$ reduction of the load-carrying capacity to $2472 \mathrm{kN}$ occurs, see Fig. $14 a$ (i). For a magnitude of $L / 125$, this reduction is as high as $54.4 \%$, see Fig. $14 a$ (ii). A significant increase in the column maximum strength is obtained for imperfection shapes in the form of Fourier sine series that assume $\delta_{1} / \delta_{2}=0.5$, corresponding to a curvature reverse of the initial bow. The figures are as follows: $24.8 \%$ reduction for an amplitude of $L / 500$ and $48.8 \%$ for $L / 125$.

3. As shown in the graphs in $b$ (i) and (ii) of Fig. 14, fixed-fixed columns are highly sensitive to the effect of initial geometric imperfections. Since the loss in the load-carrying capacity is so severe due to the imperfections, it is unlikely that the form of the imperfection itself would play a significant role.

4. Figs. 15a(i) and (ii) show the equilibrium paths for the cantilevered column (swaypermitted case). The graphs demonstrate that these columns are extremely sensitive to the initial bow amplitude and the form of the initial imperfection, with shapes affine to the first buckling mode and represented by Fourier sine series truncated after the first term being chosen. The latter representation assumes a variation of the relative basic amplitude of the imperfection selected from Table 2 with the imperfection combinations "positive" and "negative" $\delta_{0}=L / 500$ (cases 2 and 8) and $\delta_{0}=L / 125$ (cases 4 and 10) being chosen.

5. With a smaller amount of initial bow, see graph $15 a(\mathrm{i})$, the load capacity is relatively insensitive to the imperfection: the maximum load reduces to $95 \%$ of the buckling load when an initial out-of-straightness affine to the first buckling mode shape is introduced, the load reduces to $93 \%$ and $86.5 \%$ for imperfection shapes in the form of Fourier sine series (one term); the latter quantities correspond to "positive" and "negative" initial bows, respectively. This trend is not observed for larger amounts of initial bow. The graphs in Fig. 15a(ii) show that the load-carrying capacity drops to $82 \%, 93.4 \%$ and $78.6 \%$, in the same order as above. It is interesting to note that now the equilibrium path for initial bow affine to the first buckling 
mode shape almost corresponds to that of "negative" initial bow represented by Fourier sine series (one term). Another important observation relates to the "positive" initial bow case that corresponds to the column being deformed in full reversed curvature bending and renders the system less imperfection sensitive for the cases presented in the current analysis.

6. Finally, the results for the fixed-guided column are summarized in Figs. 15b(i) and (ii). Depending on the level of imperfection, and for the assumed imperfection shapes, the equilibrium responses are approximately parallel with variations in the column strength of $25 \%$ and $38 \%$ with reference to the critical load, see graph (i), or the column response can be reversed as shown in graph (ii). In this case, the range of variation in the column strength is wider, with a minimum of $56 \%$ for an initial bow affine to the lowest buckling mode and $81 \%$ for and initial bow represented by Fourier sine series (two terms) and $\delta_{1} / \delta_{2}=4$, see also Fig. 11.

\subsubsection{Discussion}

The results above use a formulation for the imperfections that are compared based on a fixed amplitude for the different functions describing the imperfections. This is perfectly reasonable for the current study for a variety of reasons. Firstly, the design codes usually give guidance on the levels of imperfection in terms of an out-of-straightness amplitude. Secondly, the Fourier series are truncated after a limited number of terms, implying that the total perturbations between different imperfection cases are of comparable size. However, in enhanced studies, where imperfections are considered with significantly smaller wavelengths, if the amplitude is fixed then the total size of the imperfection may be biased towards the smaller wavelengths due to the overall initial perturbation being intrinsically larger. An alternative measure of imperfection size has been proposed in a number of recent articles [14-16], where the inextensional end-displacement required to bend the structural element into the imperfect profile becomes the measure of imperfection size rather than the amplitude. This has the advantage of the model not being constrained and therefore able to consider a larger range of imperfection profiles. However, this consideration is left for future work and would also be readily applicable for the localized imperfections considered next.

\subsection{Localized imperfections}

In this third study, emphasis is placed on the effect of the magnitude of localized imperfections modelled as solitons. Further calculations are performed for a variety of localized and single half-sine column imperfections combinations to analyse their influence on the column behaviour. The location of the soliton is varied in both cases. The study only considers the case of pin-ended columns that represent a benchmark to which other columns with different end conditions can be referred.

\subsubsection{Parameters selected for numerical studies}

The behaviour of a pin-ended column from the basic problem is now sought with a variation in the following parameters: 
1. The amplitude of a single wave soliton, $\delta_{0, \text { sol }}$.

2. The soliton form parameter, $\varphi_{\text {sol }}$.

3. The abscissa of the soliton peak, $\xi$.

4. The initial central deflection, $\delta_{0}$.

These variables are varied parametrically as shown in Table 5. The significance of each of the above variables on the buckling load is summarized in the following section. The key results are included in Figs. 16 and 17.

\subsubsection{Principal results}

The curves in Fig. 16 pertain to the buckling characteristics of a column with localized imperfections. The two non-dimensional graphs are identical. The response is initially linear and stiff. As the load increases, the increase in displacement becomes disproportionately larger. Fig. $16 a$ also shows that:

1. An increase in the wave amplitude of the imperfection reduces the pre-buckling stiffness and the carrying capacity of the column (cases A.1 and A.4).

2. A localized imperfection closer to the column footing has a stabilizing effect (cases A.1 and A.2).

Fig. $16 b$ highlights the unfavourable effect of smaller values of the soliton form parameter $\varphi_{\text {sol. }}$. The relationship between this parameter and the load-carrying capacity is nonlinear, see also Table 5 .

As expected, the load-carrying capacity is also reduced by localized and bow imperfection combinations. The peak load and the buckling response, in general, however are relatively insensitive to the presence of such localized imperfections, as can be seen in Tables 1 and 5 and Fig. 17.

\section{Influence of end support conditions}

Different types of boundary conditions are now considered to assess the influence of the elastically restrained end supports. Results are presented for the buckling of uniform imperfect columns provided with any combination of rigid end support conditions, either symmetrical or unsymmetrical. Practical implications of the effect of sway stiffness in the column structural behaviour are also considered.

\subsection{Estimation of bounds on side sway restraints}

Consider a straight pin-ended column which is supported at the top end with a longitudinal spring (Fig. 18a). The characteristic equation for this particular column can be written as follows:

$$
\mu^{3}\left(\mu^{2}-\frac{K_{\Delta \mathrm{b}}}{E I} L\right) \sin \mu L=0
$$

where $\mu$ is given by Eq. (6). Since $\mu \neq 0$, either 


$$
\sin \mu L=0 \Leftrightarrow N_{\mathrm{cr}}=\frac{\pi^{2} E I}{L^{2}}=N_{\text {Euler }}
$$

or

$$
K_{\Delta b}=\frac{\mu^{2} E I}{L} .
$$

For $K_{\Delta \mathrm{b}}$ small, then $N_{\text {cr }}<\mathrm{N}_{\text {Euler. }}$ The maximum load, in this case is the Euler load that is attained as $K_{\Delta \mathrm{b}}$ increases. Then,

$$
K_{\Delta b, \mathrm{crit}}=\frac{\mu_{\text {Euler }}^{2} E I}{L}=\frac{\pi^{2} E I}{L^{3}}
$$

or in non-dimensional form,

$$
k_{\Delta \mathrm{b}, \mathrm{crit}}=\frac{K_{\Delta \mathrm{b}, \mathrm{crit}}}{\frac{E I}{L^{3}}}=\pi^{2} .
$$

Fig. $18 b$ shows another case where there exists a critical sway stiffness. The characteristic equation is now written as:

$$
2 \cos \mu L+\left(\mu L-\mu^{3} \frac{E I}{K_{\Delta \mathrm{b}}}\right) \sin \mu L-2=0
$$

from which

$$
\mu_{\mathrm{cr}}=\frac{2 \pi}{L} \Leftrightarrow N_{\mathrm{cr}}=\frac{4 \pi^{2} E I}{L^{2}}=4 N_{\text {Euler }}
$$

or

$$
k_{\Delta \mathrm{b}, \mathrm{crit}}=\frac{K_{\Delta \mathrm{b}, \mathrm{crit}}}{\frac{E I}{L^{3}}}=\frac{\frac{4 \pi^{2} E I}{L^{3}}}{\frac{E I}{L^{3}}}=4 \pi^{2} .
$$

The previous two examples are rather special idealized cases in that the critical load for the fully sway-prevented column is well known. The more general case of a spring-supported column at both ends is represented in Fig. 18c. For this system, the characteristic equation is given by

$$
\begin{aligned}
& -2 k_{\Delta \mathrm{b}} k_{\theta \mathrm{a}} k_{\theta \mathrm{b}}+\left[2 k_{\Delta \mathrm{b}} k_{\theta \mathrm{a}} k_{\theta \mathrm{b}}+k_{\Delta \mathrm{b}}\left(k_{\theta \mathrm{a}}+k_{\theta \mathrm{b}}\right) \mu^{2} L^{2}-\left(k_{\theta \mathrm{a}}+k_{\theta \mathrm{b}}\right) \mu^{4} L^{4}\right] \cos \mu L+ \\
& +\left[-k_{\Delta \mathrm{b}} k_{\theta \mathrm{a}}+k_{\Delta \mathrm{b}} k_{\theta \mathrm{b}}\left(k_{\theta \mathrm{a}}-1\right)-\left(k_{\Delta \mathrm{b}}+k_{\theta \mathrm{a}} k_{\theta \mathrm{b}}\right) \mu^{2} L^{2}+\mu^{4} L^{4}\right] \mu L \sin \mu L=0
\end{aligned}
$$

This equation is best solved numerically. The smallest positive root of Eq. (27) yields $\mu_{\mathrm{cr}}$ from which $N_{\text {cr }}$ can be computed. The relationship between the ratio $N_{\text {cr }} / N_{\text {Euler }}$ and the non-dimensional sway stiffness $k_{\Delta \mathrm{b}}$ is illustrated in Fig. 19, in which various rotational spring stiffness values are assumed. In Fig. $19 a$ the two rotational spring constants are equal, whereas Figs. $19 b$ and $19 c$ assume extreme values for $k_{\theta \mathrm{a}}$, corresponding to a pin end and a clamped end, respectively. Curves $\mathrm{A}$ and $\mathrm{C}$ are drawn for $k_{\theta \mathrm{b}}=0$ and $k_{\theta \mathrm{b}}=1000$, respectively. The latter value corresponds to a clamped end [23]. Curve B is drawn for a semi-rigid joint at the column top end. The graphs highlight the existence of a critical sway stiffness for some standard boundary conditions (straight vertical lines) and show the spring values from Eqs. (23) and (26). Where $k_{\theta \mathrm{a}}=k_{\theta \mathrm{b}}$ (Fig. 19a), the curves also exhibit a plateau region that commences at finite values of $k_{\Delta \mathrm{b}}$. In Figs. $19 b$ and $19 c$ the curves $N_{\mathrm{cr}} / N_{\text {Euler }}$ versus $k_{\Delta \mathrm{b}}$ are sharply curved for smaller values of $k_{\Delta \mathrm{b}}$ and are followed by a virtually straight line. 


\subsection{Parameters selected for numerical studies}

A systematic parametric study is conducted to develop a better understanding of the influence of end restraint on the strength and behaviour of columns. Table 6 shows the different parameters selected for the analysis. For comparison purposes, the sway stiffness was chosen to illustrate cases which can be considered (i) sway-prevented with effective lengths below actual length $\left(L_{\text {eff }}\right.$ is fixed to $0.75 \mathrm{~L}$ ) and (ii) sway-permitted with column effective lengths above the actual length $\left(L_{\text {eff }}\right.$ is fixed to $0.75 L$ ). Sway restraints that result in the buckling load being equal to the Euler load (i.e. $L_{\text {eff }}=L$ ) are also considered. It is important to realize from these cases that the basic sway/no-sway phenomenon depends not only on the sway stiffness value $k_{\Delta \mathrm{b}}$ but also on the rotational spring stiffness values. Table 6 shows that the sway-prevented case requires a relatively low sway stiffness for $k_{\theta \mathrm{a}} / k_{\theta \mathrm{b}} \leq 1$. Larger sway stiffness values are required in a situation whereby $k_{\theta \mathrm{a}} / k_{\theta \mathrm{b}}>1$.

In all cases, the initial bow imperfection is represented by Fourier sine series (Example 1: single term; Examples 2-7: two series terms, with $\delta_{1} / \delta_{2}=0.5,-0.5,1,-1,4,-4$, see Fig. 11). The maximum bow amplitude is maintained at $L / 500$ and the initial sway stiffness at $L / 200$.

\subsection{Principal results}

The effect of the end restraint is to increase the column stiffness and to reduce the lateral deflection with a consequent increase in strength. This is clearly illustrated in Figs. 20 and 21 that represent the typical behaviour found in the current study and Table 7 that summarizes the column load capacity factor for different end restraints.

The following trends are observed:

1. In all cases, the maximum absolute load increases as the horizontal restraining spring increases in stiffness, although the strength ratio to the critical load decreases.

2. In symmetrical cases, i.e. equal rotational spring supports at the column ends, the equilibrium paths $N_{\mathrm{Ed}} / N_{\text {cr }}$ versus $w_{\max } / L$ are practically coincident, see Fig. 20a(i)-(ii), 21a(i) and $21 b(\mathrm{i})$.

3. In unsymmetrical cases, the equilibrium paths $N_{\mathrm{Ed}} / N_{\mathrm{cr}}$ versus $w_{\max } / L$ do not overlap, see Figs. 20b-c, $21 a$ (ii) and $21 b$ (ii). In these graphs, the upper curves correspond to the swayprevented situation. The two other curves are very closely related.

4. The usual approach of using the concept of the column effective length to account for end restraint does not give a good approximation of the strength of a column with initial bow. Consider $L_{\text {eff }}=1$ (cases $i .2$ in Table 7, the pinned-end and fixed-guided ends in Table 1, for example). In all cases, the initial bow amplitude is equal to $L / 500$. Depending on the form of the bow imperfection and the end restraining conditions, the strength ratio to the buckling load varies from 0.67 (pinned-ends and imperfection affine to the first buckling mode) to $0.84\left(k_{\theta \mathrm{a}}=5, k_{\theta \mathrm{b}}=1\right.$ and the imperfection is represented by two Fourier sine series with $\left.\delta_{1} / \delta_{2}=0.5\right)$. This represents a $20 \%$ variation in the load-carrying capacity. 


\section{Conclusions and further developments}

The current work presents an appraisal of the effects of the magnitude, shape and orientation of equivalent initial geometric imperfections on the load-deflection response of an otherwise straight column. The approach for imperfection sensitivity studies deals directly with the imperfect configuration and includes imperfection shapes that are not consistent with the kinematic boundary conditions of the column. The system is modelled with a generalized imperfection $w_{0}$, which is formulated from equilibrium. This generalized imperfection accounts for all other geometric imperfections and the unavoidable eccentricity of the axial load. Localized imperfections are also included in the analysis to estimate the column strength and the load-deflection behaviour. The influence of end restraint on column strength is also taken into account since columns in actual frameworks are connected to other structural members such that their ends are restrained.

In examining the effect of the imperfection shape and amplitude on a column with arbitrary end restraining conditions, the following points have been noted:

1. The pre-buckling stiffness and the load-carrying capacity of the column are generally reduced by increasing the amplitude of the imperfection.

2. The imperfection shape again changes the pre-buckling stiffness and the load-carrying capacity of the column.

3. The critical load degradation is not necessarily more severe when the imperfection shape is affine to the first buckling mode.

4. Current analyses show that there is no obvious relationship between the form of the imperfection and the column maximum strength.

5. The effect of a single local imperfection is to reduce the column strength; this effect however becomes much less when combined with a general column imperfection.

6. The imperfection sensitivity to the end-restraining conditions (end rotational fixities and side sway restraint) has been clearly demonstrated. The effects of a wide range of initial geometrical imperfection shapes on the equilibrium response have been evaluated through parametric studies. The results show that the usual approach of using the concept of the column effective length to account for end restraint does not give a good approximation of the column strength.

7. In the case of sway-permitted columns with rotationally rigid connections, with initial bow amplitudes larger than $L / 500$ can cause reversals in the lateral deflections. This behaviour is also very much dependent on the form of the imperfection.

The practical implications of the above results clearly deserve further careful study. Additionally, the determination of the critical (or worst) column imperfection, i.e. the imperfection shape that decreases the column strength more rapidly, remains open despite the contributions of this work. 


\section{Appendix A}

Consider the pin-ended column with initial sway imperfection $\Delta_{0}$ and initial bow given by the first buckling mode shape (Fig. A1):

$$
w_{0}(x)=\frac{\Delta_{0}}{L} x+\delta_{0} \sin \frac{\pi x}{L}
$$

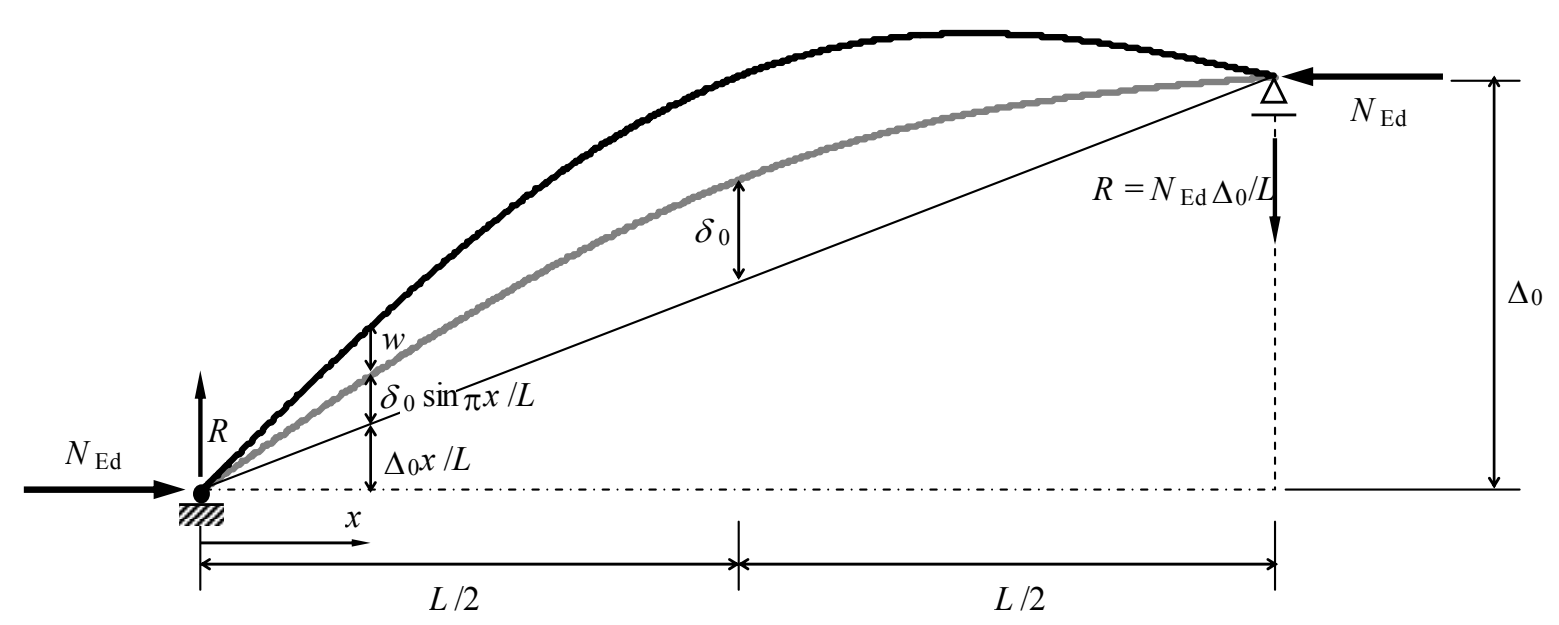

Fig. A1. Pin-ended column with initial imperfections.

The bending moment at a distance $x$ from the support is:

$$
M=-N_{\mathrm{Ed}}\left[\Delta_{0}-\left(\frac{\Delta_{0}}{L} x+\delta_{0} \sin \frac{\pi x}{L}+w\right)\right]+R(L-x)
$$

and:

$$
R=N_{\mathrm{Ed}} \frac{\Delta_{0}}{L}
$$

The internal bending moment is related to the curvature by means of Eq. (4). The elimination of $M$ from this equation and Eq. (A2) provides the following differential equation in $w$ :

$$
-E I \frac{d^{2} w}{d x^{2}}-N_{\mathrm{Ed}} w=N_{\mathrm{Ed}}\left[-\Delta_{0}+\frac{\Delta_{0}}{L} x+\delta_{0} \sin \frac{\pi x}{L}+\frac{\Delta_{0}}{L}(L-x)\right]
$$

that is more conveniently written as:

$$
w^{\prime \prime}+\mu^{2} w=-\mu^{2} \delta_{0} \sin \frac{\pi x}{L}
$$

This equation is independent from the initial sway displacement $\Delta_{0}$. 


\section{References}

[1] Euler L. Methodus inveniendi lineas curvas maximi minimive proprietate gaudentes. Appendix: De curvis elasticis. Lausanne and Geneva; 1744.

[2] Timoshenko SP, Gere JM. Theory of Elastic Stability. Singapore: McGraw-Hill; 1961.

[3] Simitses GJ. An Introduction to the Elastic Stability of Structures. Malabar, Florida: Robert E. Krieger Publishing Company; 1976.

[4] Chen WF, Atsuta T. Theory of Beam-Columns. Volume 1: In-plane behavior and design. New York: McGraw-Hill; 1976.

[5] Timoshenko SP. History of strength of materials. New York: McGraw-Hill; 1953.

[6] American Institute of Steel Construction (AISC). Specification for structural steel buildings. ANSI/AISC Standard No. 360-10. Chicago; 2010.

[7] European Committee for Standardization (CEN). Eurocode 3: Design of steel structures - Part 1-1: General rules and rules for buildings. EN 1993-1-1. Brussels; 2005.

[8] European Committee for Standardization (CEN). Execution of steel structures and aluminium structures - Part 2: Technical requirements for steel structures. EN 1990-2. Brussels; 2008.

[9] Boyce WE. Buckling of a column with random initial displacements. J Aerosp Sci 1961; 28(4): 308-313.

[10] Fraser WB, Budiansky B. The buckling of a column with random initial deflections. J Appl Mech 1969; 36(2): 233-240.

[11] Ayrton WE, Perry J. On struts. The Engineer 1886; 62: 464.

[12] Robertson A. The strength of struts. Proc ICE, selected engineering paper $n^{\circ} .28 ; 1925$.

[13] Rondal J, Maquoi R. Single equation for SSRC column strength curves. J Struct Div. ASCE 1979; 105(ST1): 247-250.

[14] Wadee MA. Effects of periodic and localized imperfections on struts on nonlinear foundations and compression sandwich panels. Int. J. Solids Struct. 2000; 37, 1191-1209.

[15] Wadee MA, Simões da Silva LAP. Asymmetric secondary buckling in monosymmetric sandwich struts. ASME J Appl Mech 2005; 72: 683-690.

[16] Saito D and Wadee MA. Buckling behaviour of prestressed steel stayed columns with imperfections and stress limitation. Eng Struct 2009; 31: 1-15.

[17] Chen WF, Lui EM. Structural stability: theory and implementation. New York: Prentice Hall; 1987.

[18] Natanson IP. Constructive Function Theory. Volume I - Uniform Approximation. New York: Frederick Ungar Publishing Co; 1964.

[19] Apostol TM. Calculus. Volume II: Multi-variable calculus and linear algebra, with applications to differential equations and probability. USA: John Wiley \& Sons; 1969.

[20] Wolfram. Mathematica 8, Produced by Wolfram Corp., Champaign; 2010.

[21] Bailey WN. Generalized hypergeometric series. Cambridge University Press; 1935.

[22] Hirt MA, Bez R. Construction métallique - Notions fondamentales et methods de dimensionnement. Traité de Génie Civil de l'Ecole polytechnique fédérale de Lausanne. Lausanne: Presses polytechniques et universitaires romandes; 1994.

[23] Simão PD, Girão Coelho AM, Bijlaard FSK. Influence of splices on the buckling of columns. Int J Non-Linear Mech 2012; 47: 806-822. 
Table 1.

Critical loads $N_{\mathrm{cr}}$ and strength ratios (normalized with $N_{\mathrm{cr}}$ ) for different imperfection levels.

\begin{tabular}{lrrrrr}
\hline \multicolumn{1}{c}{ Column end-restraints } & \multirow{2}{*}{$N_{\text {cr }}(\mathrm{kN})$} & \multicolumn{4}{c}{ Initial bow amplitude $\delta_{0}$} \\
& & $L / 1000$ & $L / 500$ & $L / 250$ & \multicolumn{1}{c}{$L / 125$} \\
\hline Pinned-ends & 3546.5 & 0.75 & 0.67 & 0.56 & 0.45 \\
Fully fixed & 14586.0 & 0.24 & 0.23 & 0.21 & 0.18 \\
Fixed-free & 911.6 & 0.97 & 0.95 & 0.90 & 0.82 \\
Fixed-guided & 3546.5 & 0.82 & 0.75 & 0.67 & 0.56 \\
\hline
\end{tabular}

Table 2.

Numerical examples and parametric variations in sway imperfection and maximum initial bow amplitude.

\begin{tabular}{cccccc}
\hline Example & $\Delta_{0}$ & $\delta_{0}$ & Example & $\Delta_{0}$ & $\delta_{0}$ \\
\hline 1 & $L / 200$ & $L / 1000$ & 7 & $L / 200$ & $-L / 1000$ \\
2 & $L / 200$ & $L / 500$ & 8 & $L / 200$ & $-L / 500$ \\
3 & $L / 200$ & $L / 250$ & 9 & $L / 200$ & $-L / 250$ \\
4 & $L / 200$ & $L / 125$ & 10 & $L / 200$ & $-L / 125$ \\
5 & $L / 500$ & $L / 1000$ & 11 & $L / 500$ & $-L / 1000$ \\
6 & $L / 500$ & $L / 125$ & 12 & $L / 500$ & $-L / 125$ \\
\hline
\end{tabular}

Table 3.

Strength ratios to the critical load: summary of results.

\begin{tabular}{ccccc}
\hline \multirow{2}{*}{ Example } & \multicolumn{4}{c}{ Column end-restraints } \\
& Pinned-ends & Fully fixed & Fixed-free & Fixed-guided \\
\hline 1 & 0.75 & 0.24 & 0.91 & 0.64 \\
2 & 0.67 & 0.23 & 0.93 & 0.62 \\
3 & 0.56 & 0.20 & 0.98 & 0.59 \\
4 & 0.45 & 0.17 & 0.93 & 0.62 \\
5 & 0.75 & 0.24 & 0.98 & 0.75 \\
6 & 0.45 & 0.17 & 0.88 & 0.75 \\
7 & 0.75 & 0.24 & 0.88 & 0.64 \\
8 & 0.67 & 0.23 & 0.86 & 0.62 \\
9 & 0.56 & 0.20 & 0.84 & 0.59 \\
10 & 0.45 & 0.17 & 0.79 & 0.53 \\
11 & 0.75 & 0.24 & 0.94 & 0.75 \\
12 & 0.45 & 0.17 & 0.83 & 0.60 \\
\hline
\end{tabular}


Table 4.

Strength ratios to the critical load: summary of results for different column end restraints.

\begin{tabular}{|c|c|c|c|c|c|c|c|c|c|c|c|c|}
\hline \multirow[t]{2}{*}{ Ex. } & \multicolumn{4}{|c|}{$\begin{array}{l}\text { Pinned-ends } \\
\text { Ratio } \delta_{1} / \delta_{2}\end{array}$} & \multicolumn{8}{|c|}{$\begin{array}{l}\text { Fixed-guided ends } \\
\text { Ratio } \delta_{1} / \delta_{2}\end{array}$} \\
\hline & \pm 0.5 & \pm 1 & \pm 2 & \pm 4 & 0.5 & 1 & 2 & 4 & -0.5 & -1 & -2 & -4 \\
\hline 1 & 0.83 & 0.80 & 0.78 & 0.76 & 0.68 & 0.67 & 0.66 & 0.65 & 0.62 & 0.63 & 0.63 & 0.63 \\
\hline 2 & 0.75 & 0.72 & 0.70 & 0.68 & 0.71 & 0.68 & 0.66 & 0.64 & 0.60 & 0.60 & 0.61 & 0.61 \\
\hline 3 & 0.64 & 0.62 & 0.59 & 0.57 & 0.80 & 0.90 & 0.77 & 0.62 & 0.55 & 0.56 & 0.57 & 0.57 \\
\hline 4 & 0.51 & 0.49 & 0.47 & 0.46 & 0.60 & 0.65 & 0.73 & 0.79 & 0.48 & 0.50 & 0.51 & 0.51 \\
\hline 5 & 0.83 & 0.80 & 0.78 & 0.76 & 0.85 & 0.82 & 0.79 & 0.77 & 0.71 & 0.72 & 0.73 & 0.73 \\
\hline 6 & 0.51 & 0.49 & 0.47 & 0.46 & 0.56 & 0.60 & 0.65 & 0.68 & 0.51 & 0.53 & 0.55 & 0.56 \\
\hline 7 & 0.83 & 0.80 & 0.78 & 0.76 & 0.62 & 0.63 & 0.63 & 0.63 & 0.68 & 0.67 & 0.66 & 0.65 \\
\hline 8 & 0.75 & 0.72 & 0.70 & 0.68 & 0.60 & 0.60 & 0.61 & 0.61 & 0.71 & 0.68 & 0.66 & 0.64 \\
\hline 9 & 0.64 & 0.62 & 0.59 & 0.57 & 0.55 & 0.56 & 0.57 & 0.57 & 0.80 & 0.73 & 0.66 & 0.62 \\
\hline 10 & 0.51 & 0.49 & 0.47 & 0.46 & 0.48 & 0.50 & 0.51 & 0.51 & 0.60 & 0.65 & 0.73 & 0.58 \\
\hline 11 & 0.83 & 0.80 & 0.78 & 0.76 & 0.71 & 0.72 & 0.73 & 0.73 & 0.85 & 0.82 & 0.79 & 0.77 \\
\hline 12 & 0.51 & 0.49 & 0.47 & 0.46 & 0.51 & 0.53 & 0.55 & 0.56 & 0.56 & 0.60 & 0.65 & 0.73 \\
\hline
\end{tabular}


Table 5.

Numerical examples for different soliton parameters.

\begin{tabular}{|c|c|c|c|c|c|c|c|}
\hline Example & Case & $\varphi_{\text {sol }}$ & $\delta_{0, \mathrm{sol}}$ & $\xi$ & $\delta_{0}$ & Initial shape & $N_{\max } / N_{\mathrm{cr}}$ \\
\hline \multirow{6}{*}{ A } & A. 1 & \multirow{3}{*}{40} & \multirow{3}{*}{$L / 500$} & $0.25 L$ & - & & 0.84 \\
\hline & A. 2 & & & $0.50 L$ & - & & 0.82 \\
\hline & A. 3 & & & $0.75 L$ & - & & 0.84 \\
\hline & A. 4 & \multirow{3}{*}{40} & \multirow{3}{*}{$L / 1000$} & $0.25 L$ & - & & 0.91 \\
\hline & A. 5 & & & $0.50 L$ & - & & 0.89 \\
\hline & A. 6 & & & $0.75 L$ & - & & 0.91 \\
\hline \multirow{3}{*}{ B } & B.1 & \multirow{3}{*}{80} & \multirow{3}{*}{$L / 500$} & $0.25 \mathrm{~L}$ & - & & 0.85 \\
\hline & B. 2 & & & $0.50 L$ & - & & 0.84 \\
\hline & B.3 & & & $0.75 L$ & - & & 0.85 \\
\hline \multirow{3}{*}{$\mathrm{C}$} & C. 1 & \multirow{3}{*}{160} & \multirow{3}{*}{$L / 500$} & $0.25 L$ & - & & 0.86 \\
\hline & C. 2 & & & $0.50 L$ & - & & 0.85 \\
\hline & C. 3 & & & $0.75 L$ & & & 0.86 \\
\hline \multirow{3}{*}{$\mathrm{D}$} & D.1 & \multirow{3}{*}{20} & \multirow{3}{*}{$L / 500$} & $0.25 L$ & - & & 0.81 \\
\hline & D. 2 & & & $0.50 L$ & - & & 0.78 \\
\hline & D. 3 & & & $0.75 L$ & - & & 0.81 \\
\hline
\end{tabular}


Table 5.

(cont'd.)

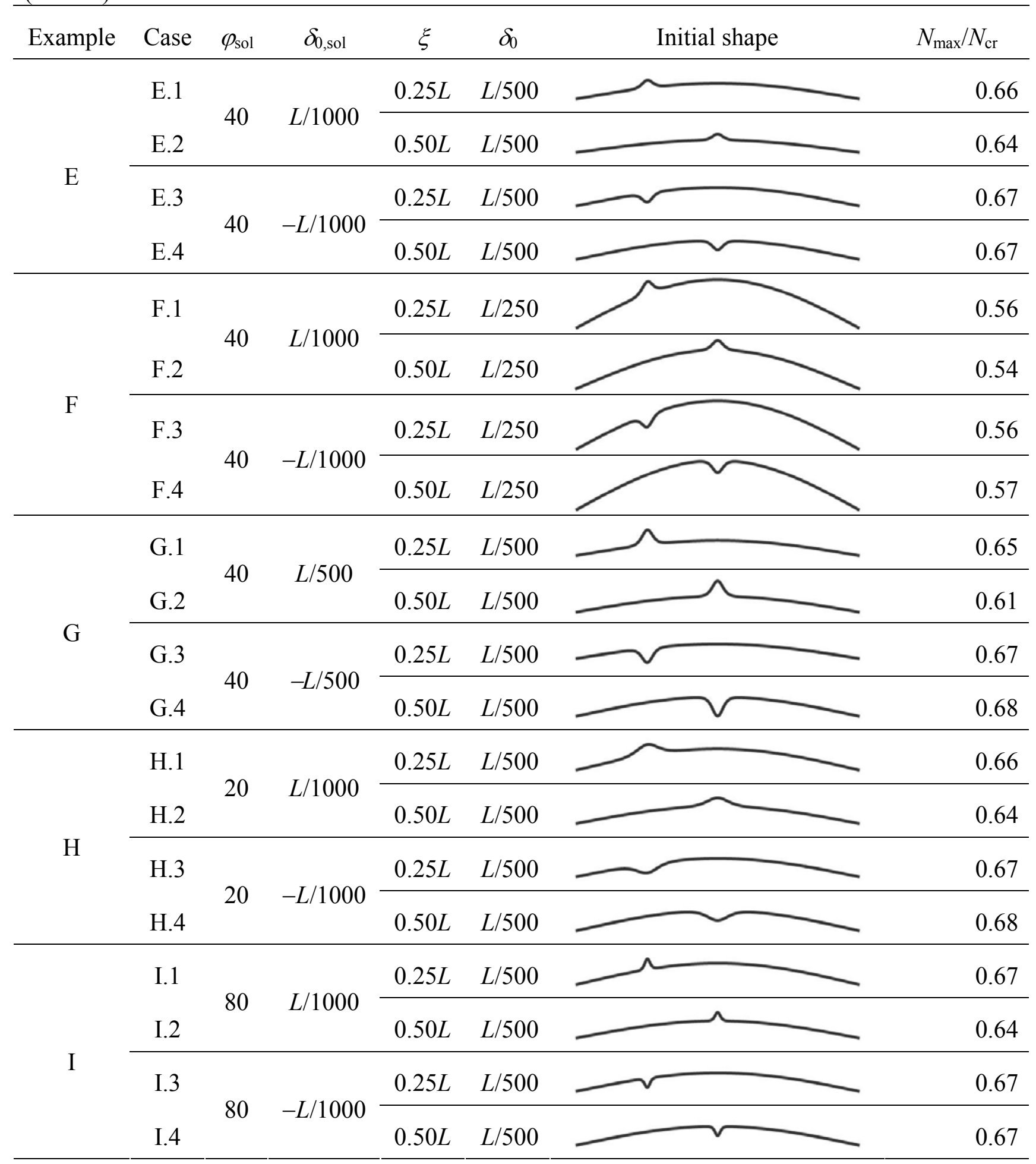


Table 6.

Numerical examples and parametric variations in elastic end-restraints.

\begin{tabular}{|c|c|c|c|c|c|c|c|}
\hline Case & & & $k_{\theta \mathrm{a}}$ & $k_{\theta \mathrm{b}}$ & $k_{\Delta \mathrm{b}}$ & $L_{\text {eff }} / L$ & $N_{\mathrm{cr}}(\mathrm{kN})$ \\
\hline \multirow{3}{*}{1} & 1.1 & Sway-prevented & 5 & 5 & 13.12 & 0.75 & 6482.7 \\
\hline & 1.2 & & 5 & 5 & 4.90 & 1 & 3646.5 \\
\hline & 1.3 & Sway-permitted & 5 & 5 & 1.15 & 1.25 & 2333.8 \\
\hline \multirow{3}{*}{2} & 2.1 & Sway-prevented & 5 & 1 & 344.99 & 0.75 & 6482.7 \\
\hline & 2.2 & & 5 & 1 & 7.38 & 1 & 3646.5 \\
\hline & 2.3 & Sway-permitted & 5 & 1 & 3.46 & 1.25 & 2333.8 \\
\hline \multirow{3}{*}{3} & 3.1 & Sway-prevented & 5 & 10 & 12.68 & 0.75 & 6482.7 \\
\hline & 3.2 & & 5 & 10 & 4.20 & 1 & 3646.5 \\
\hline & 3.3 & Sway-permitted & 5 & 10 & 0.36 & 1.25 & 2333.8 \\
\hline \multirow{3}{*}{4} & 4.1 & Sway-prevented & 1 & 5 & 344.99 & 0.75 & 6482.7 \\
\hline & 4.2 & & 1 & 5 & 7.38 & 1 & 3646.5 \\
\hline & 4.3 & Sway-permitted & 1 & 5 & 3.46 & 1.25 & 2333.8 \\
\hline \multirow{3}{*}{5} & 5.1 & Sway-prevented & 10 & 5 & 12.68 & 0.75 & 6482.7 \\
\hline & 5.2 & & 10 & 5 & 4.20 & 1 & 3646.5 \\
\hline & 5.3 & Sway-permitted & 10 & 5 & 0.36 & 1.25 & 2333.8 \\
\hline
\end{tabular}


Table 7.

Strength ratios to the critical load: summary of results.

\begin{tabular}{|c|c|c|c|c|c|c|c|c|c|}
\hline & Ex. 1 & Ex. 2 & Ex. 3 & Ex. 4 & Ex. 5 & Ex. 6 & Ex. 7 & Max. variation \\
\hline & & \multicolumn{8}{|c|}{ Initial shape } \\
\hline \multicolumn{2}{|c|}{ Case } & \multicolumn{8}{|c|}{$N_{\max } / N_{\mathrm{cr}}$} \\
\hline \multirow{3}{*}{1} & 1.1 & 0.47 & 0.52 & 0.47 & 0.52 & 0.47 & 0.50 & 0.47 & $9.6 \%$ \\
\hline & 1.2 & 0.70 & 0.74 & 0.70 & 0.72 & 0.70 & 0.70 & 0.70 & $5.4 \%$ \\
\hline & 1.3 & 0.80 & 0.84 & 0.79 & 0.83 & 0.79 & 0.81 & 0.80 & $5.9 \%$ \\
\hline \multirow[t]{2}{*}{2} & 2.2 & 0.75 & 0.84 & 0.74 & 0.81 & 0.75 & 0.76 & 0.76 & $11.9 \%$ \\
\hline & 2.3 & 0.87 & 0.88 & 0.84 & 0.87 & 0.85 & 0.87 & 0.86 & $4.5 \%$ \\
\hline \multirow{3}{*}{3} & 3.1 & 0.49 & 0.51 & 0.46 & 0.51 & 0.47 & 0.50 & 0.47 & $9.8 \%$ \\
\hline & 3.2 & 0.68 & 0.73 & 0.68 & 0.71 & 0.68 & 0.69 & 0.68 & $6.8 \%$ \\
\hline & 3.3 & 0.78 & 0.84 & 0.76 & 0.82 & 0.77 & 0.80 & 0.78 & $9.5 \%$ \\
\hline \multirow{3}{*}{5} & 5.1 & 0.50 & 0.54 & 0.47 & 0.54 & 0.47 & 0.52 & 0.47 & $13.0 \%$ \\
\hline & 5.2 & 0.68 & 0.73 & 0.67 & 0.71 & 0.68 & 0.69 & 0.68 & $8.2 \%$ \\
\hline & 5.3 & 0.79 & 0.83 & 0.76 & 0.82 & 0.76 & 0.80 & 0.78 & $8.4 \%$ \\
\hline
\end{tabular}




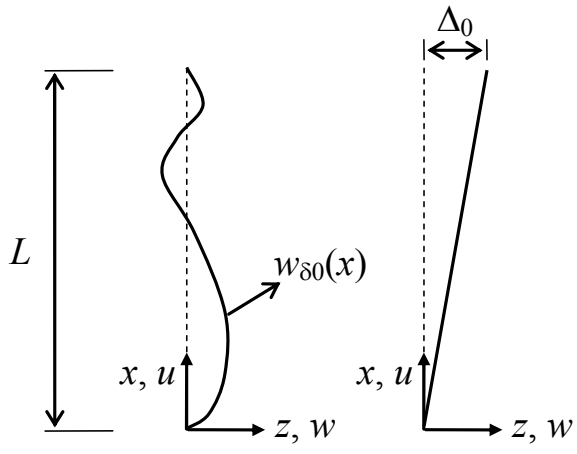

Initial geometric imperfections

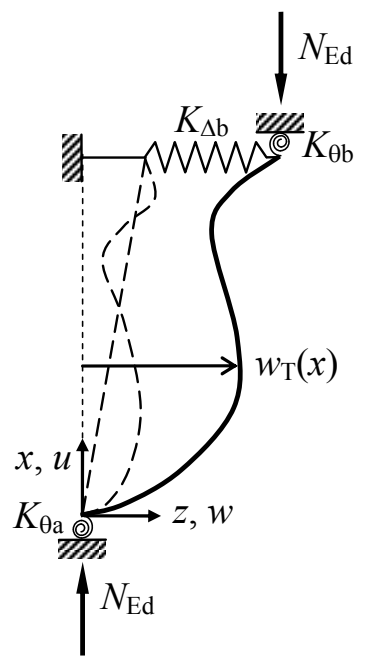

Lateral deflections of the axially loaded column

Fig. 1. Elastically supported column with equivalent geometric imperfections.

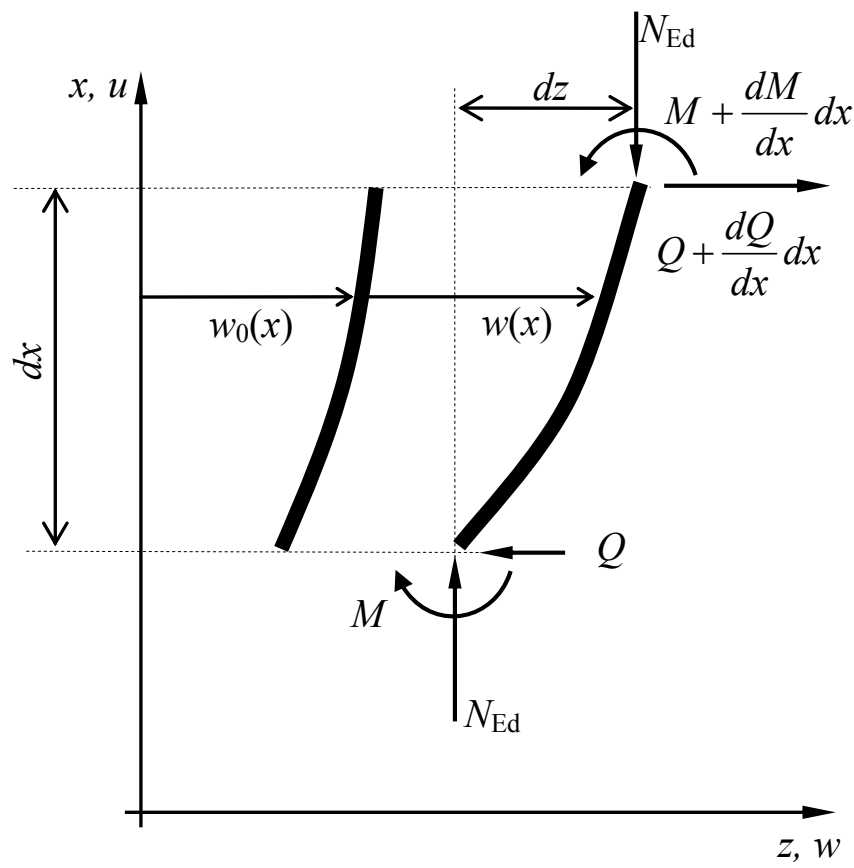

Fig. 2. Free-body diagram of a column segment. 


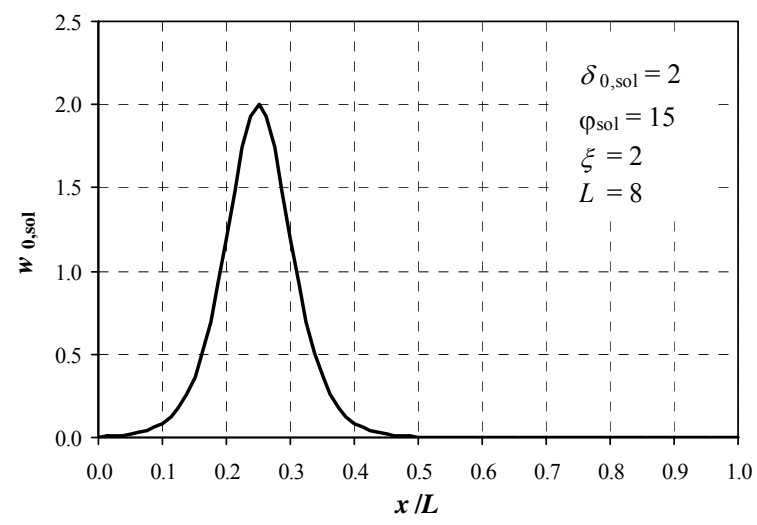

Fig. 3. Form of localized imperfection $w_{0, \text { sol }}$.

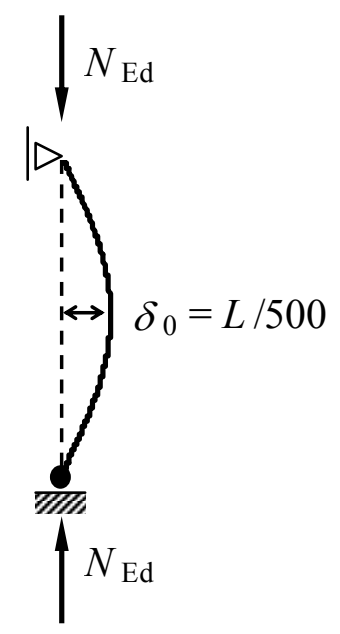

$$
\begin{aligned}
& w_{0}=w_{\delta 0}=\delta_{0} \sin \left(\frac{\pi x}{L}\right) \\
& \delta_{0}=\frac{L}{500} \text { (geometrical tolerance to EN 1090) }
\end{aligned}
$$

Fig. 4. Summary of the basic problem.

(a)

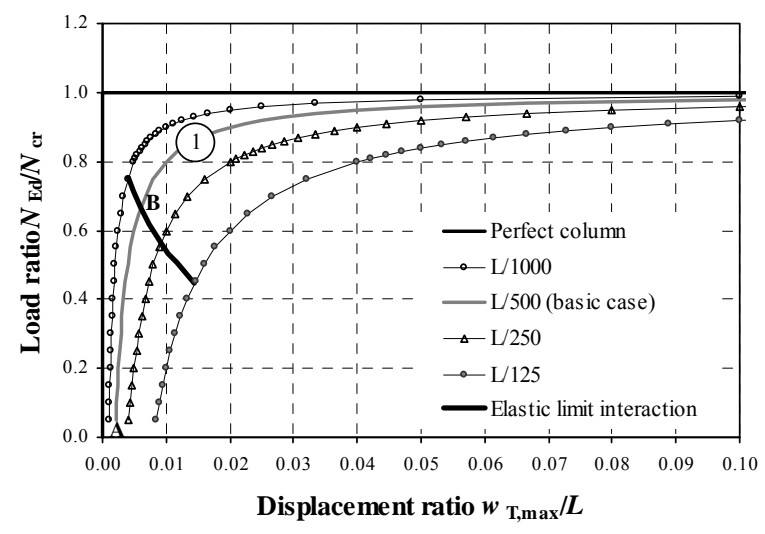

(b)

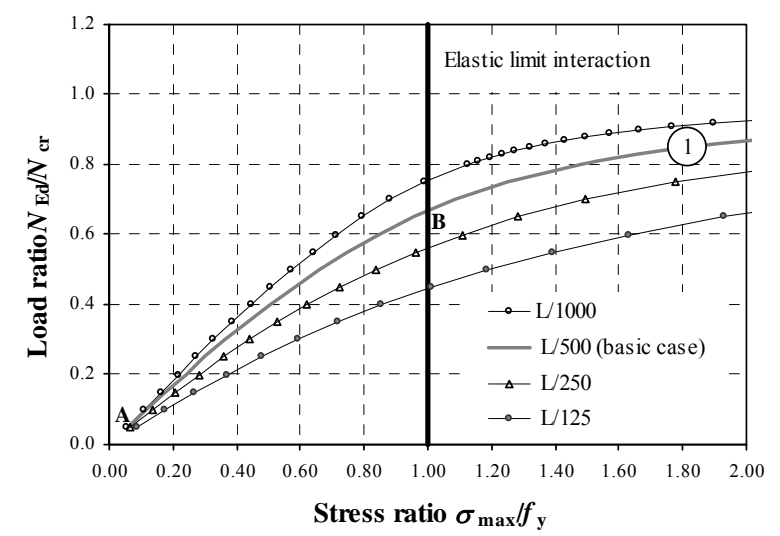

Fig. 5. Axially loaded pin-ended column with various amounts of initial bow. (a) Load versus total transverse displacement. (b) Load versus critical stress. 

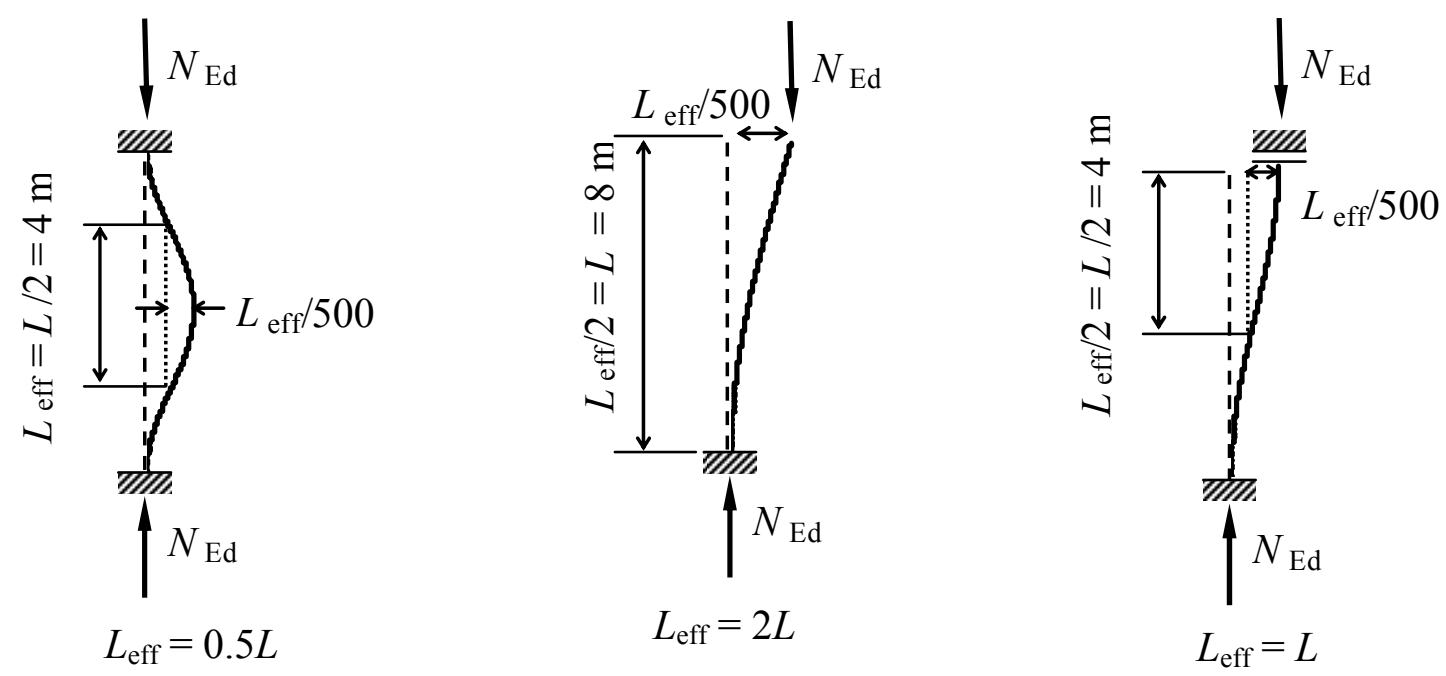

Fig. 6. Equivalent pin-ended columns with initial bow affine to the first mode shape.

(a)

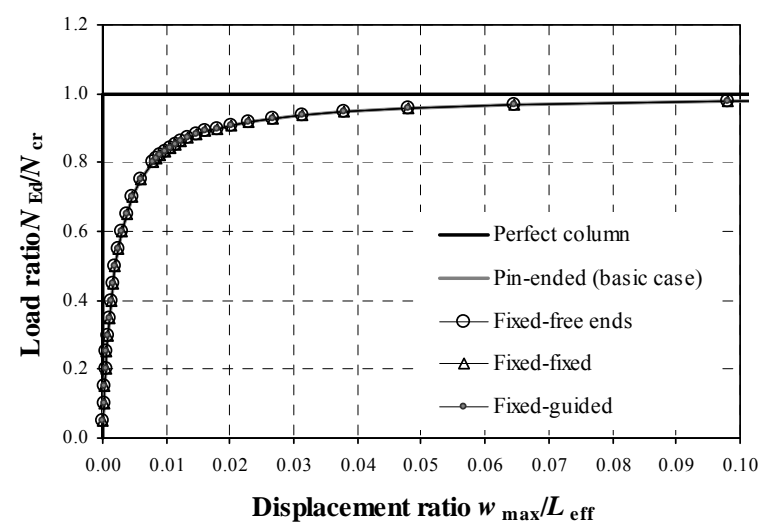

(c)

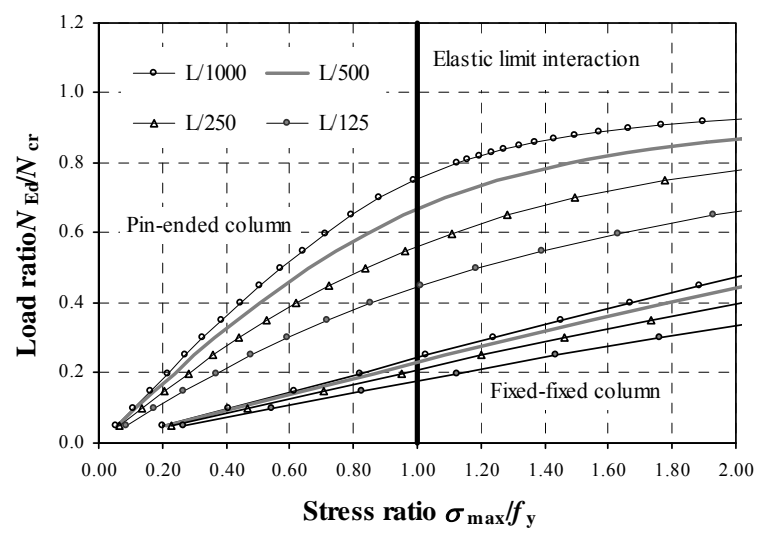

(b)

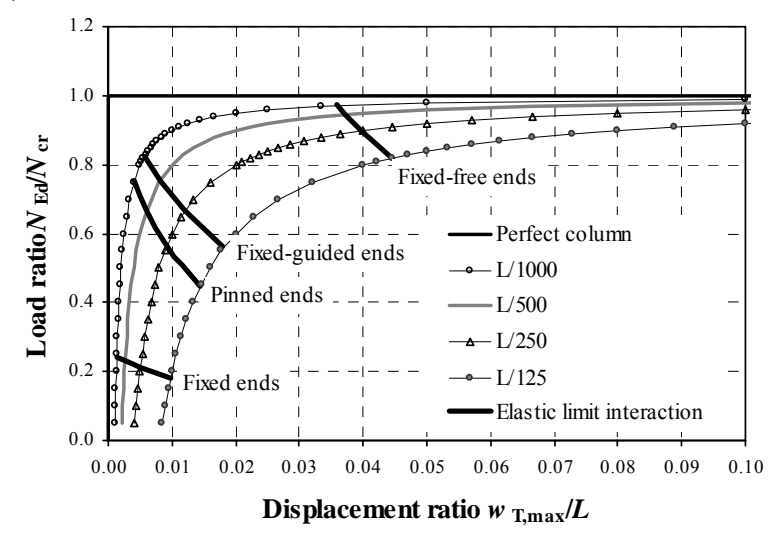

(d)

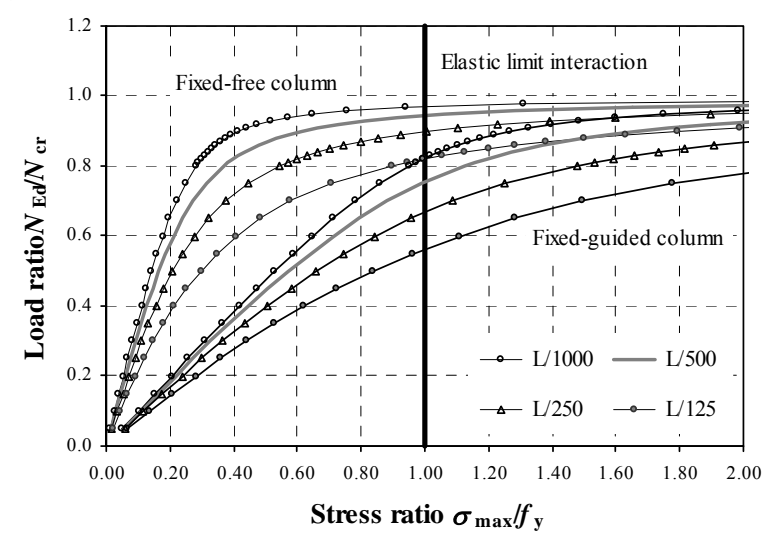

Fig. 7. Axially loaded columns with various amounts of initial bow. (a) and (b) Load versus transverse displacement. (c) Load versus critical stress: columns without sway. (d) Load vs. critical stress: columns with sway. 
(a)

(b)
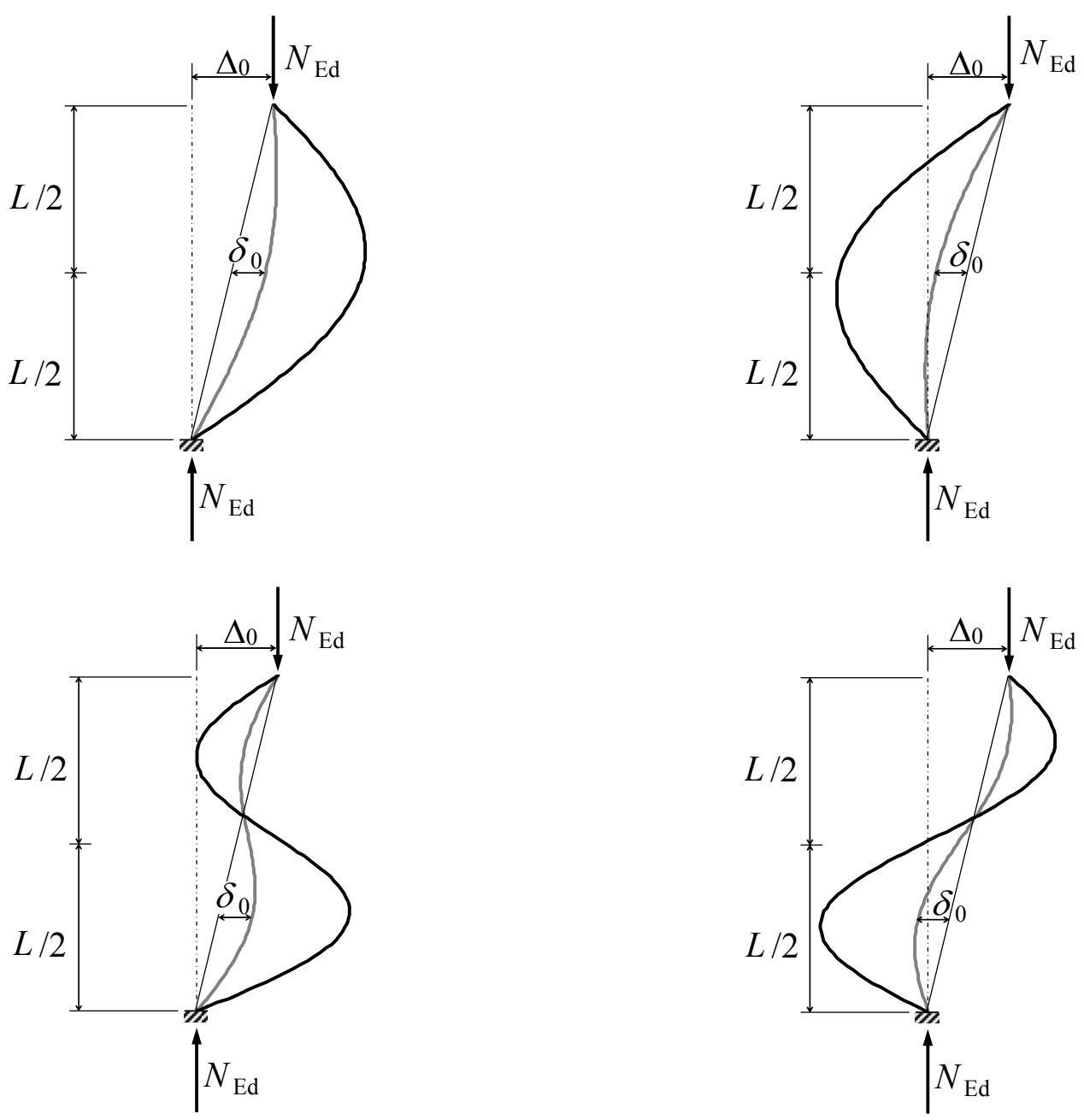

Fig. 8. Combination of initial imperfections with increasing initial bow amplitudes: positive $\Delta_{0}$ and $\delta_{0}$; positive $\Delta_{0}$ and negative $\delta_{0}$. (a) Fourier sine series truncated at $n=1$. (b) Fourier sine series truncated at $n=2$ and $\delta_{1}=0.5 \delta_{2}$ (e.g. cantilevered column).

(a)

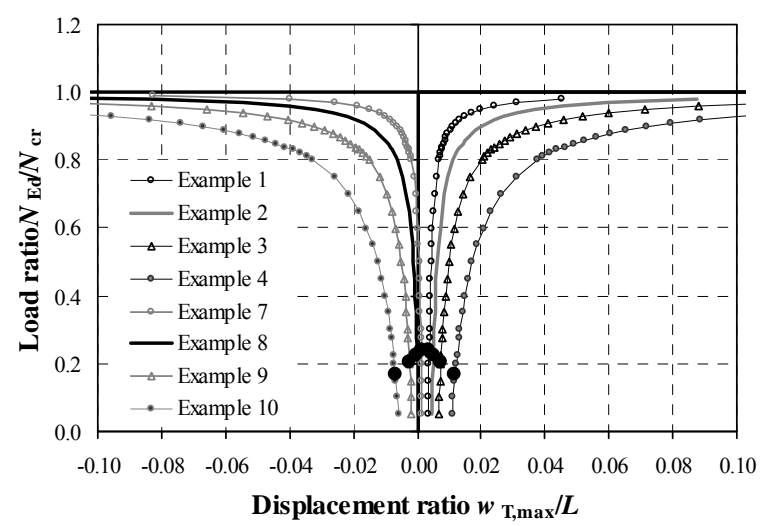

(b)

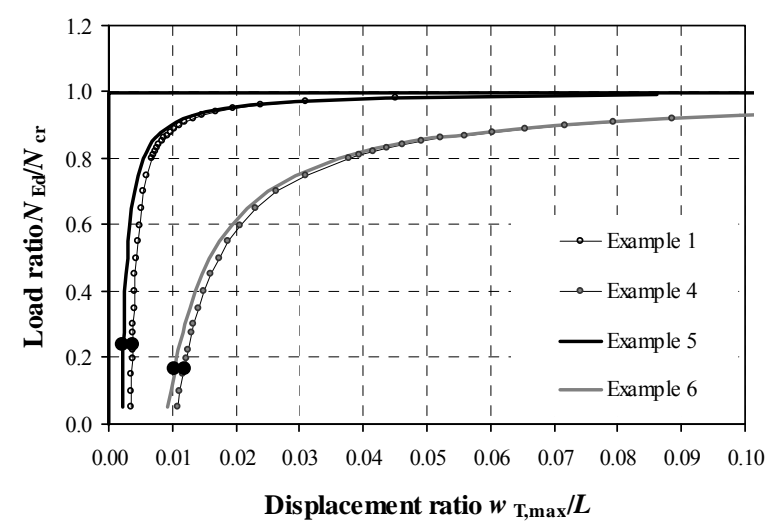

Fig. 9. Axially loaded sway-prevented (fixed-fixed) column with various amounts of initial bow.

(a) Load vs. maximum total transverse displacement for $\Delta_{0}=L / 200$. (b) Comparisons for different sway displacements. 
(i)

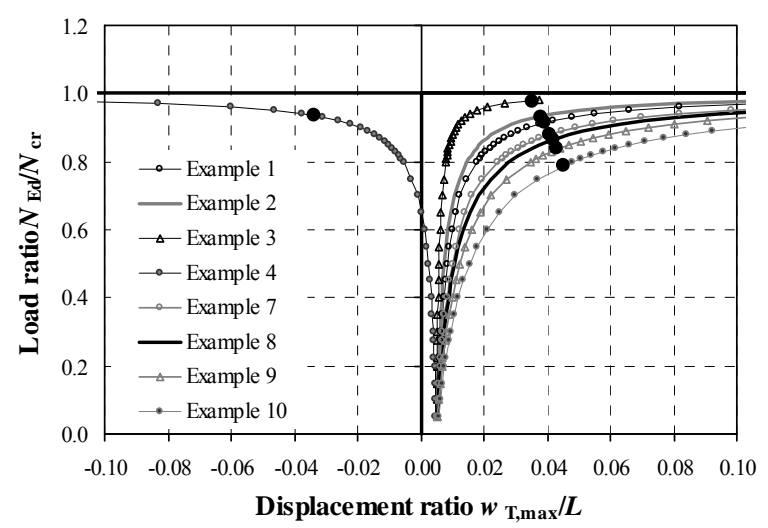

(b)

(i)

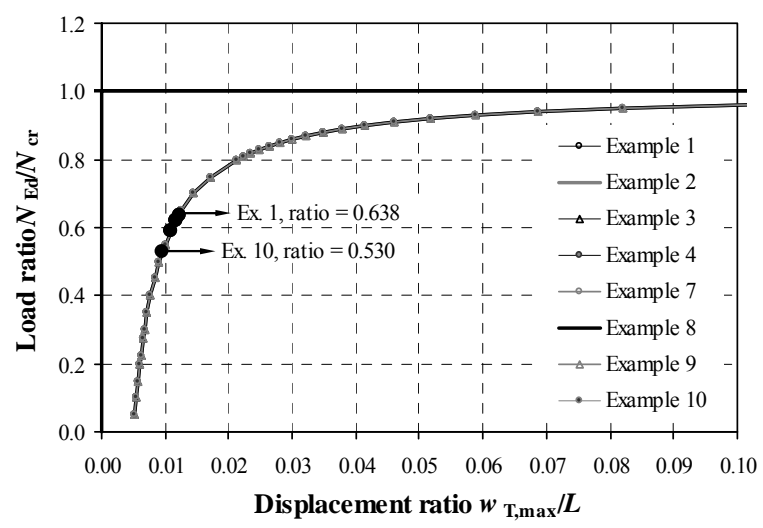

(ii)

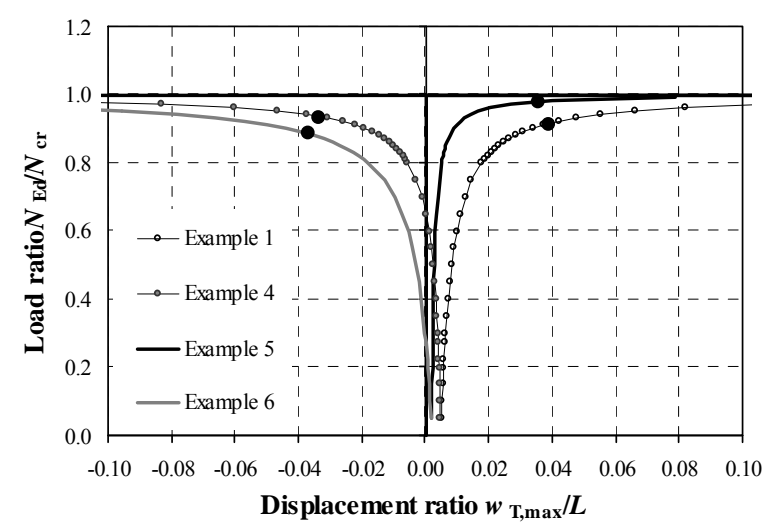

(ii)

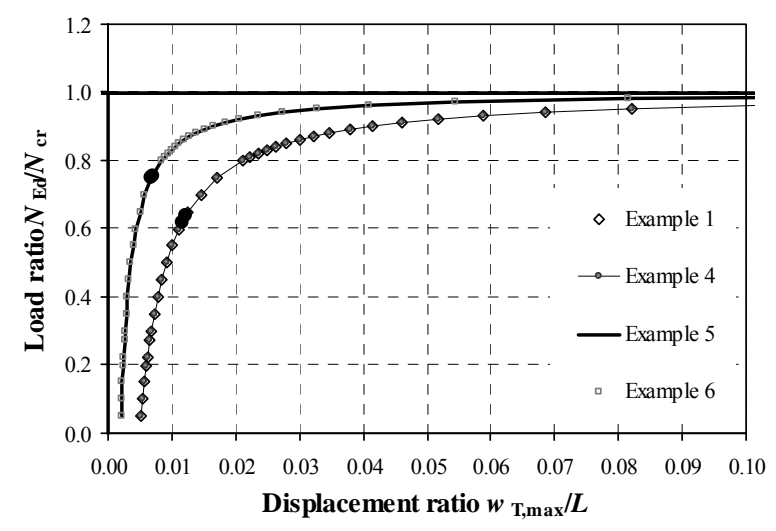

Fig. 10. Axially loaded sway-permitted column with various amounts of initial bow. (a) Cantilevered column (i) Load $v$ s. maximum total transverse displacement for $\Delta_{0}=L / 200$; (ii) Comparisons for different sway displacements. (b) Fixed-guided column (i) Load vs. maximum total transverse displacement for $\Delta_{0}=L / 200$; (ii) Comparisons for different sway displacements.

(a)

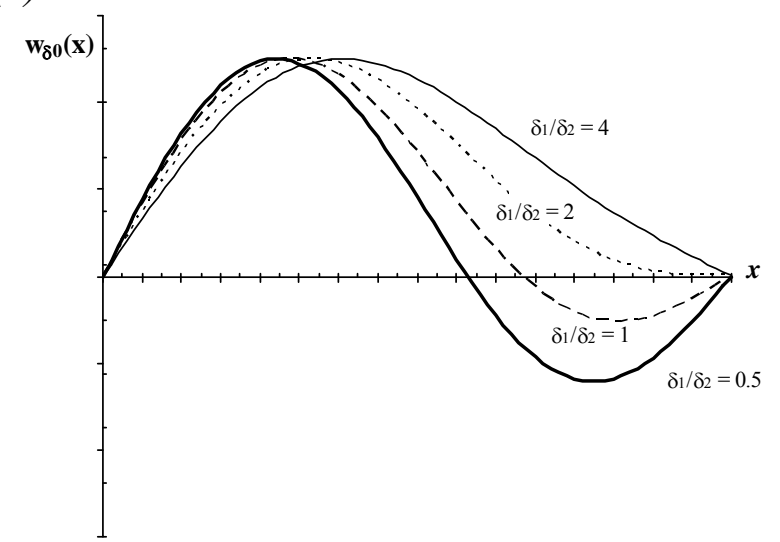

(b)

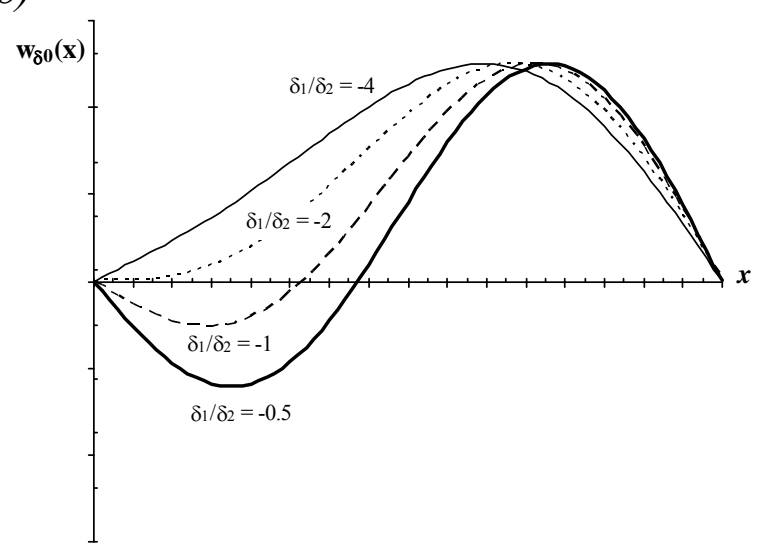

Fig. 11. Initial imperfection shapes represented a Fourier sine series with $n=2$. 
(a)

(i)

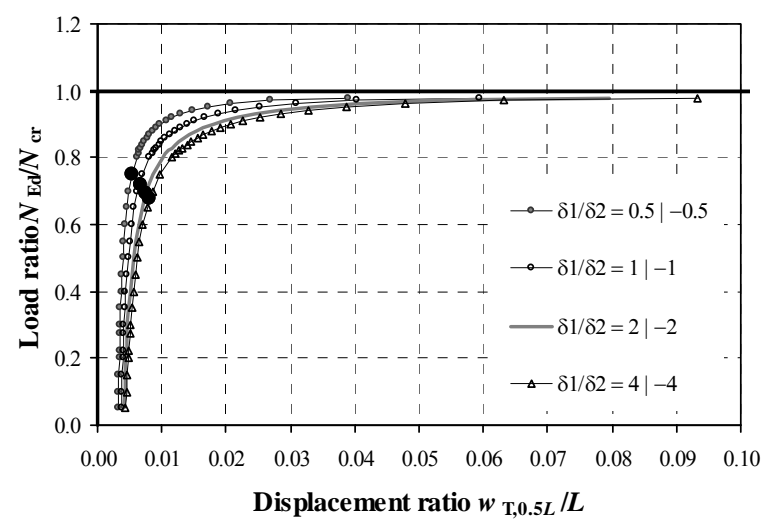

(b)

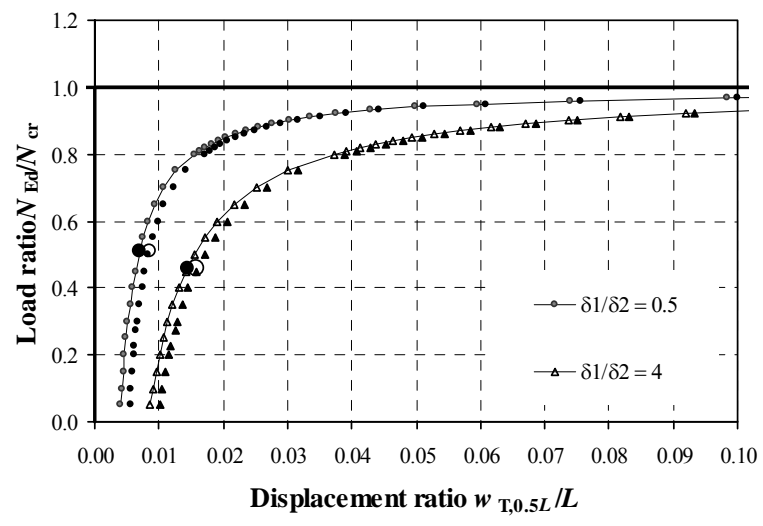

(ii)

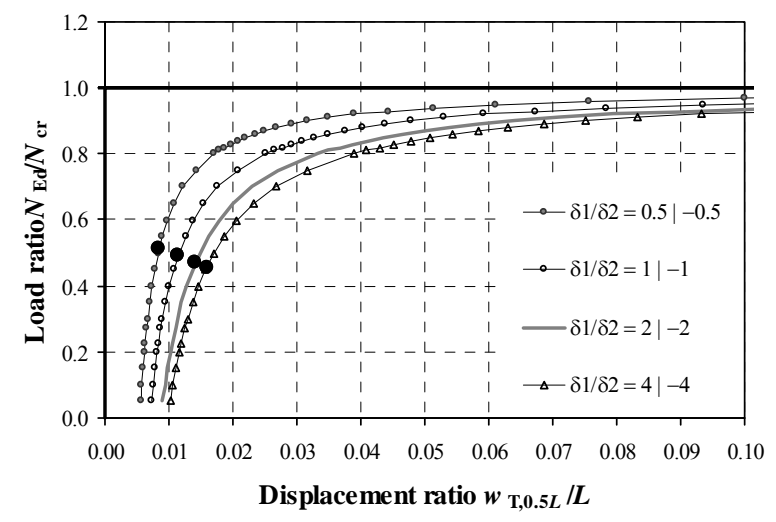

Fig. 12. Typical load-deflection curves for pin-ended columns. (a) Load vs. total transverse displacement at column mid-height (i) Example $2\left(\delta_{0}=L / 500\right)$; (ii) Example $4\left(\delta_{0}=L / 125\right)$. (b) Comparisons for different sway displacements (examples 4 and $6, \delta_{0}=L / 125, \Delta_{0}=L / 200$ and $\Delta_{0}=$ $L / 500$, respectively). 
(a)

(i)

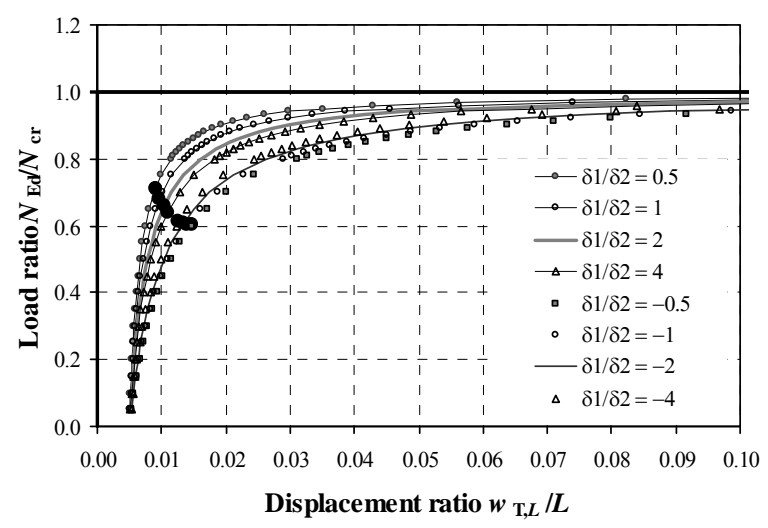

(b)

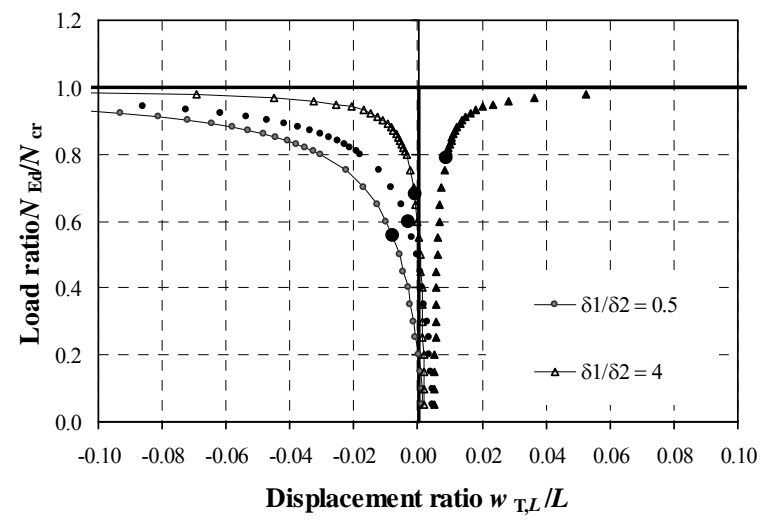

(ii)

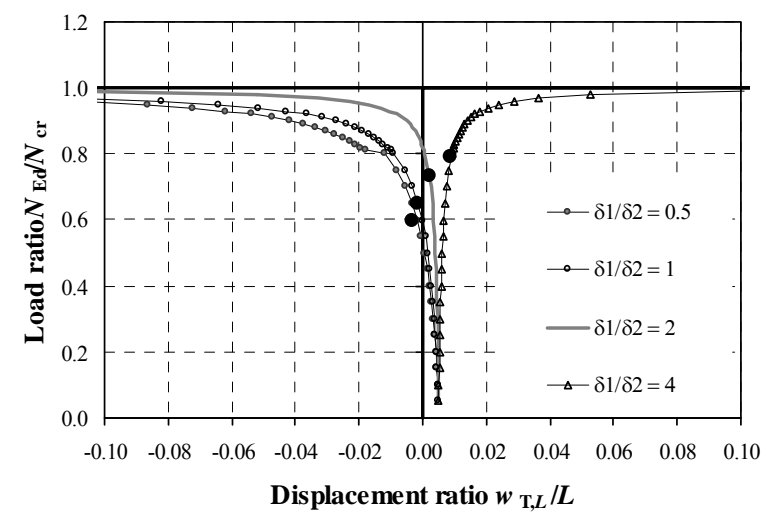

Fig. 13. Typical load-deflection curves for fixed-guided columns. (a) Load vs. total transverse displacement at the column top (i) Example $2\left(\delta_{0}=L / 500\right)$; (ii) Example $4\left(\delta_{0}=L / 125\right)$. (b)

Comparisons for different sway displacements (examples 4 and $6, \delta_{0}=L / 125, \Delta_{0}=L / 200$ and $\Delta_{0}=$ $L / 500$, respectively). 
(a)

(i)

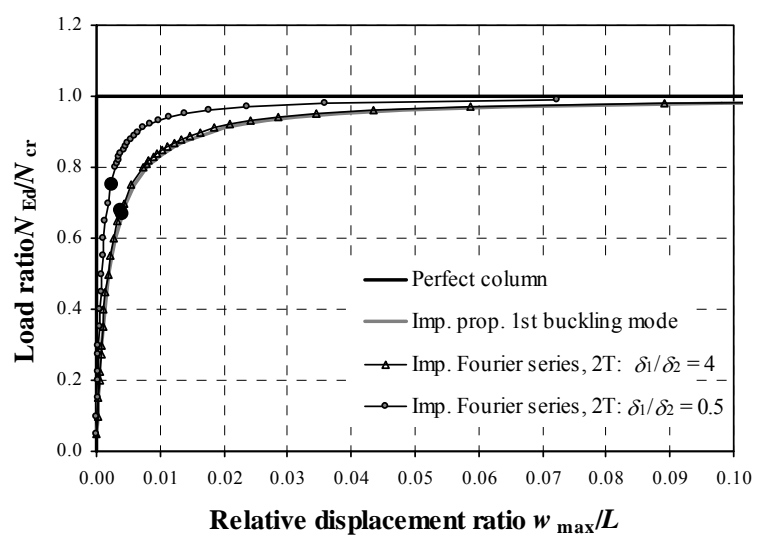

(b)

(i)

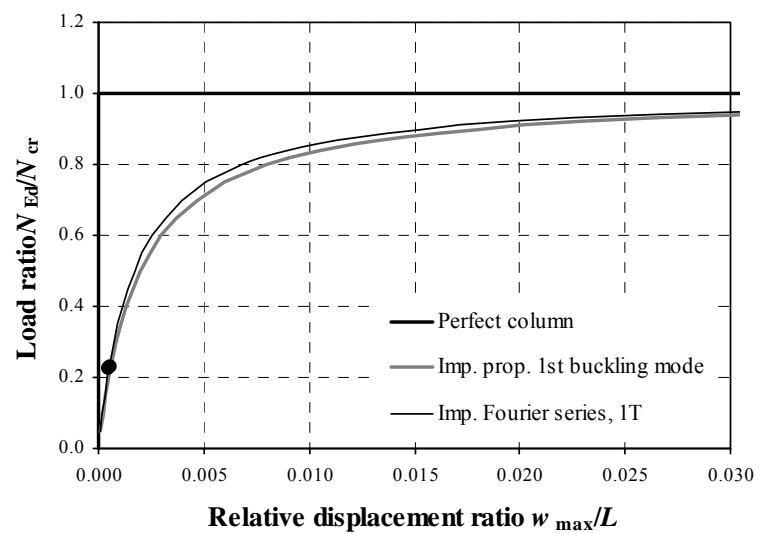

(ii)

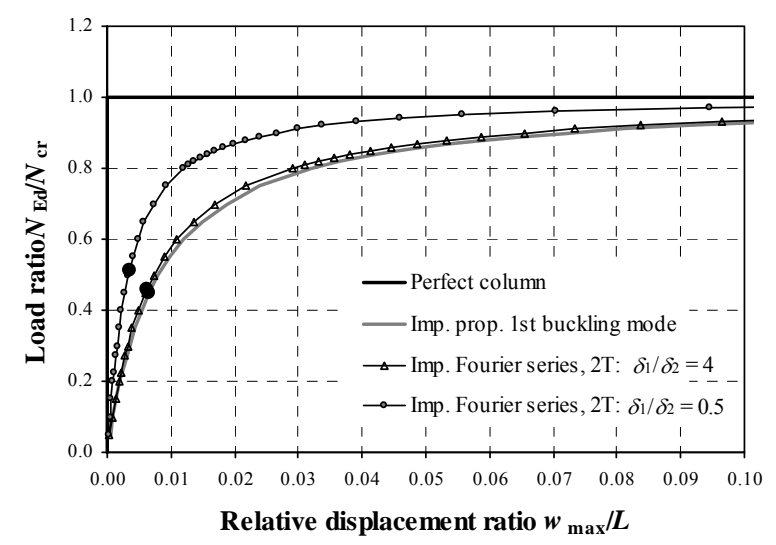

(ii)

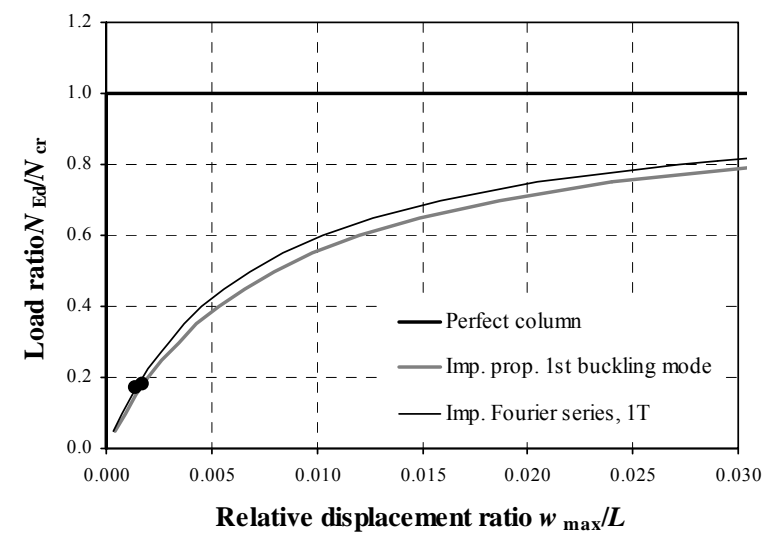

Fig. 14. Axially loaded sway-prevented columns. (a) Pin-ended column (i) $\delta_{0}=L / 500$; (ii) $\delta_{0}=$ $L / 125$. (b) Fixed-fixed column (i) $\delta_{0}=L / 500$; (ii) $\delta_{0}=L / 125$. 
(a)

(i)

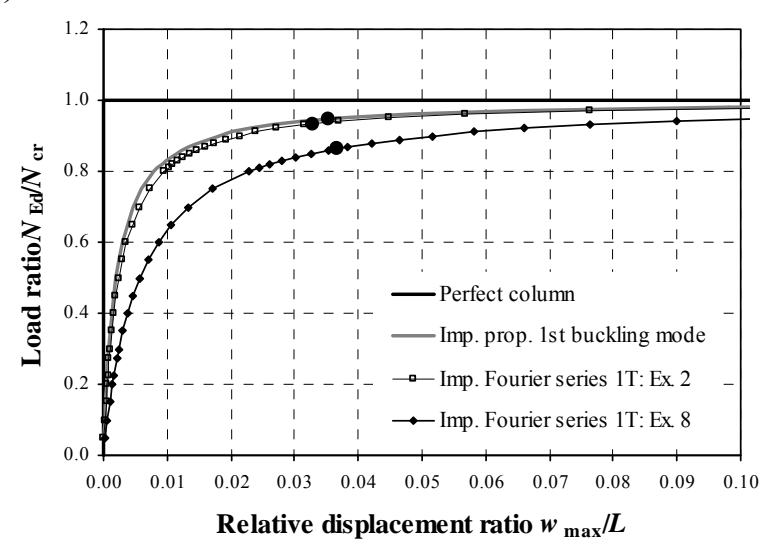

(b)

(i)

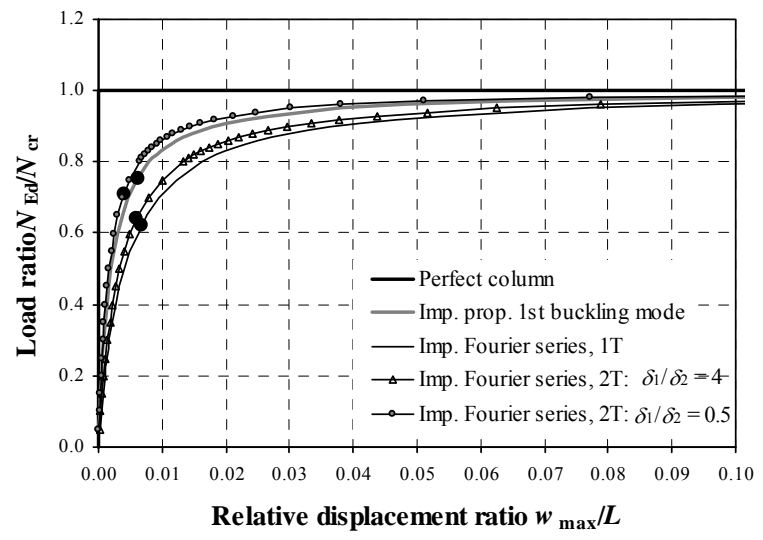

(ii)

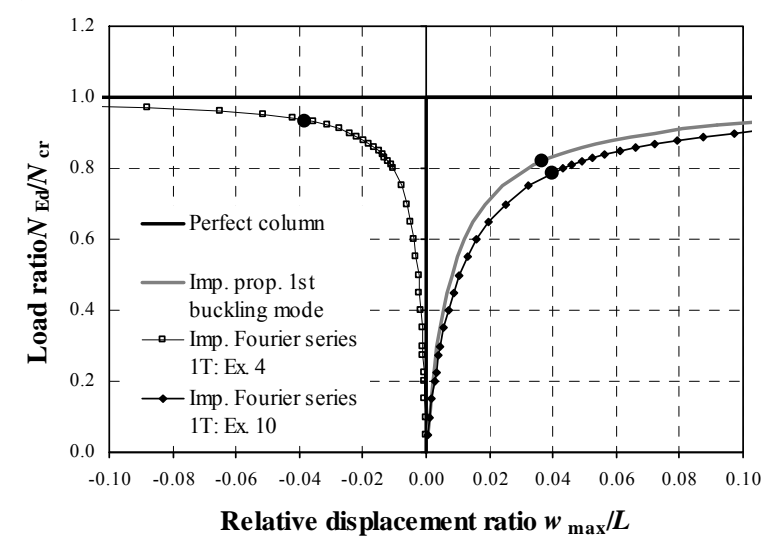

(ii)

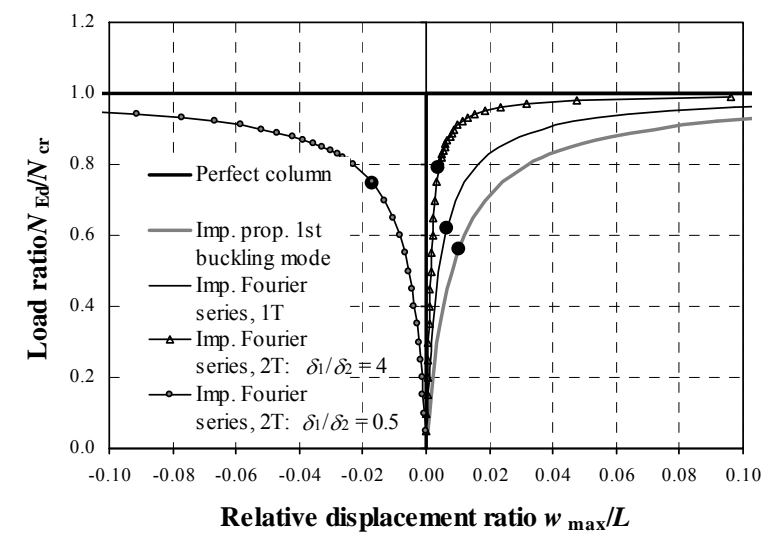

Fig. 15. Axially loaded sway-permitted columns. (a) Cantilevered column (i) $\delta_{0}= \pm L / 500$; (ii) $\delta_{0}=$ $\pm L / 125$. (b) Fixed-guided column (i) $\delta_{0}=L / 500$; (ii) $\delta_{0}=L / 125$.

(a)

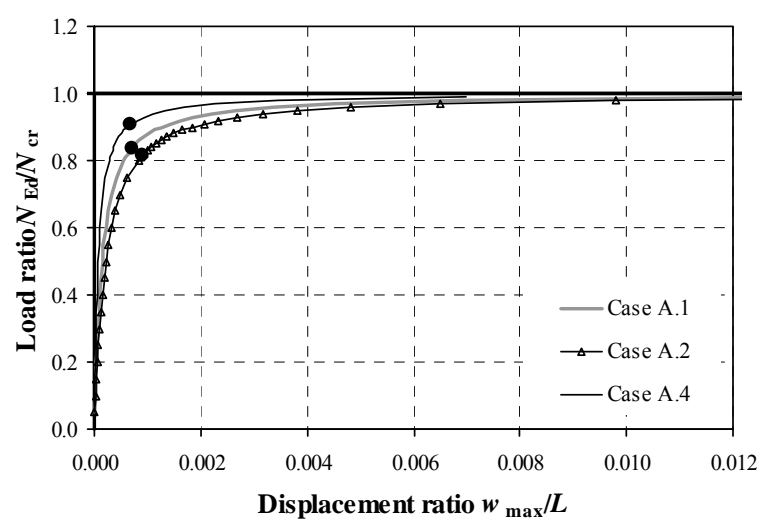

(b)

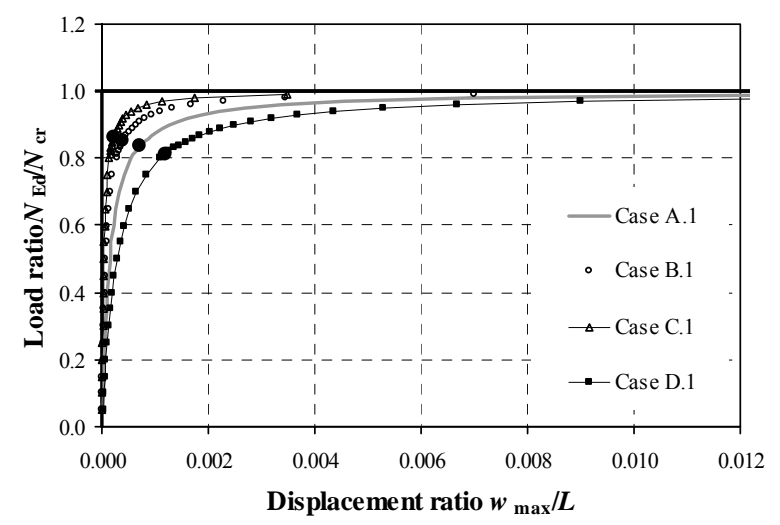

Fig. 16. Typical load-deflection curves for pin-ended columns with local imperfections modelled as solitons. (a) Effect of the wave amplitude and location. (b) Effect of form parameter $\varphi_{\text {sol. }}$. 
(a)

(i)

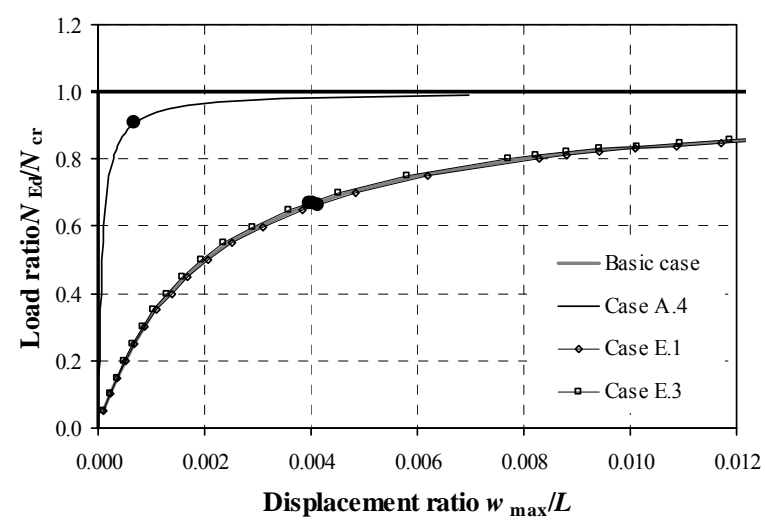

(b)

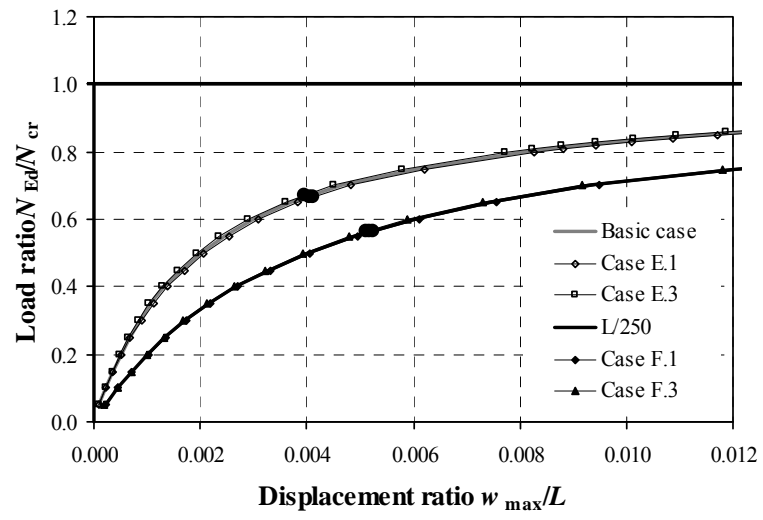

(ii)

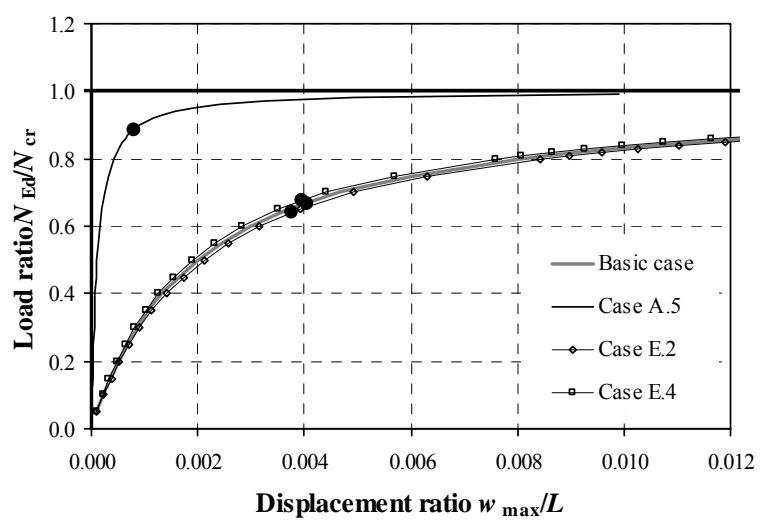

(c)

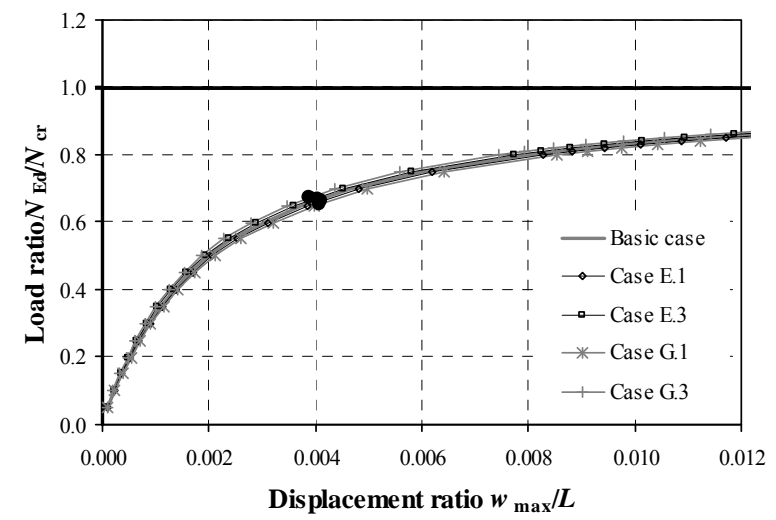

Fig. 17. Typical load-deflection curves for pin-ended columns with combined localized and single half-sine imperfections. (a) Effect of the location of the soliton peak (i) $0.25 \mathrm{~L}$; (ii) $0.50 \mathrm{~L}$. (b) Effect of initial bow amplitude and soliton orientation. (c) Effect of the soliton wave amplitude and orientation.
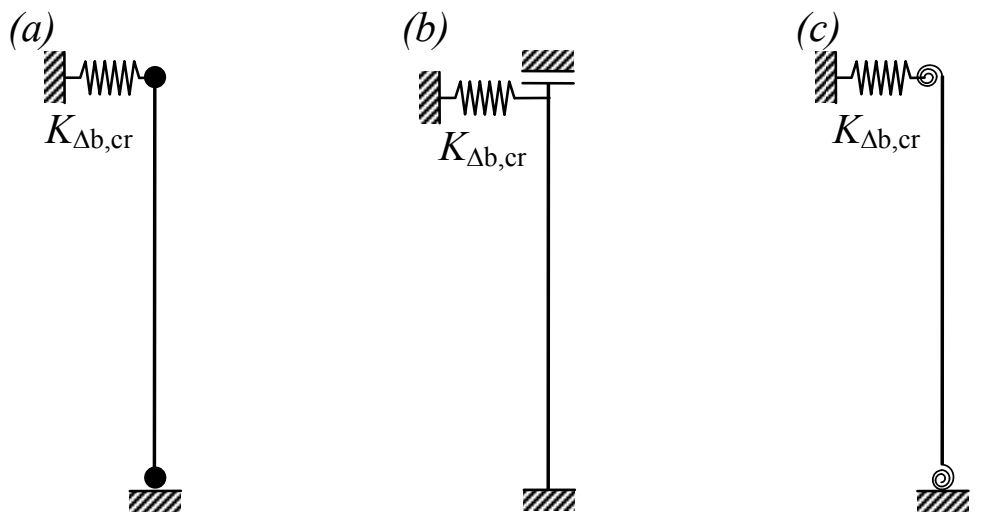

Fig. 18. Critical spring stiffness model. (a) Pinned ends. (b) Rigid ends. (c) Elastically restrained ends. 
(a)

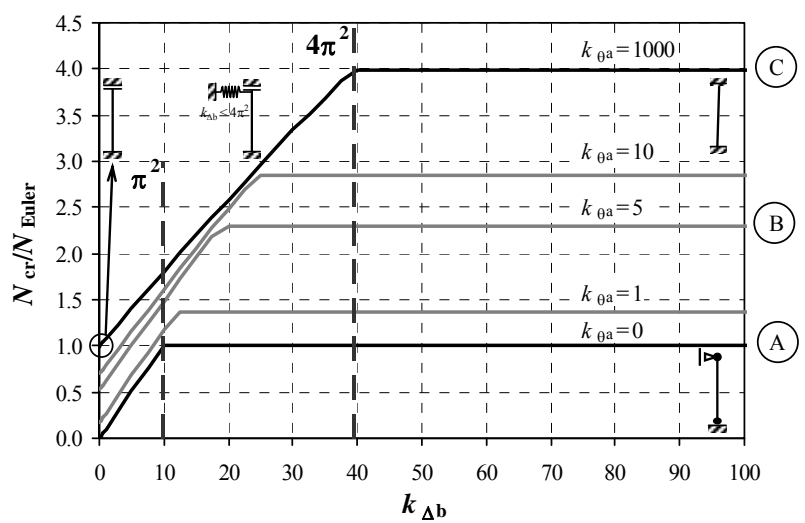

(b)

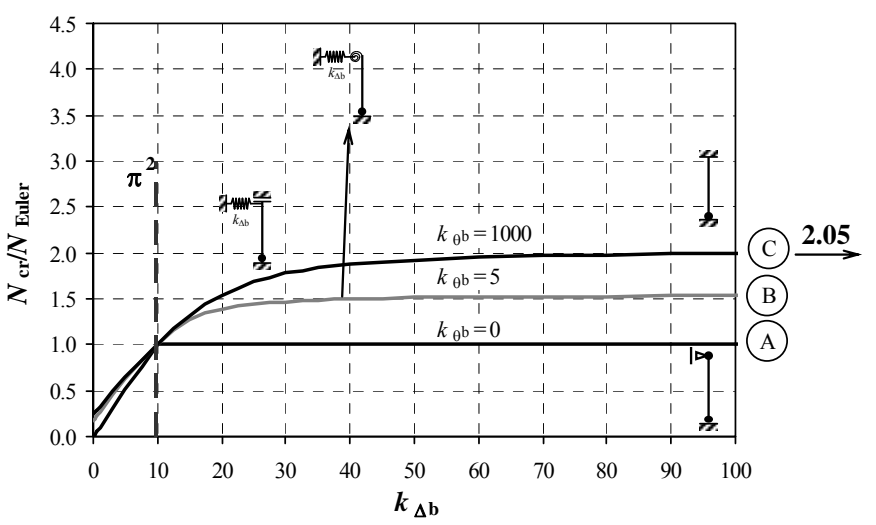

(c)

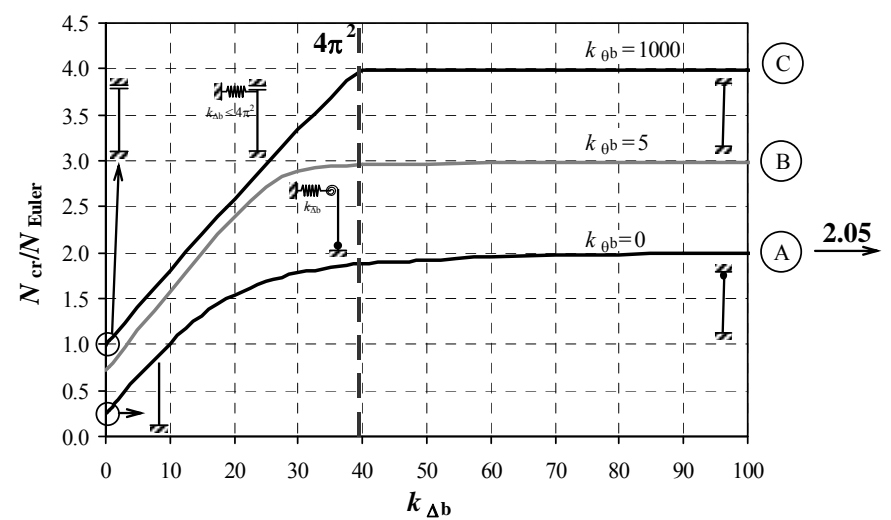

Fig. 19. Typical curves demonstrating the transition from sway-permitted columns to partly sway and nearly fully sway-prevented. (a) $k_{\theta \mathrm{a}}=k_{\theta \mathrm{b}}$. (b) $k_{\theta \mathrm{a}}=0$. (c) $k_{\theta \mathrm{a}}=\infty$. 
(a)

(i)

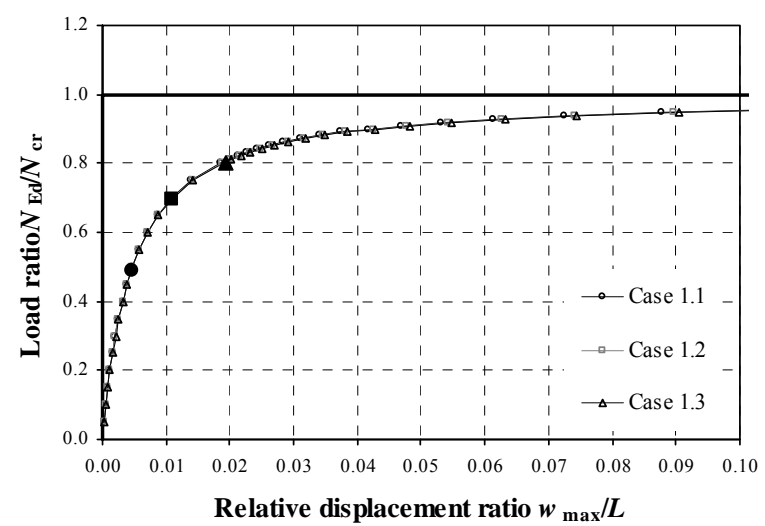

(b)

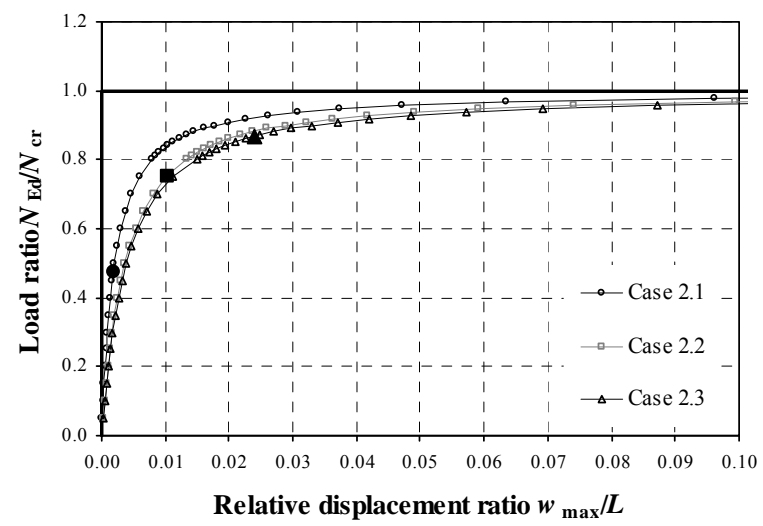

(d)

(i)

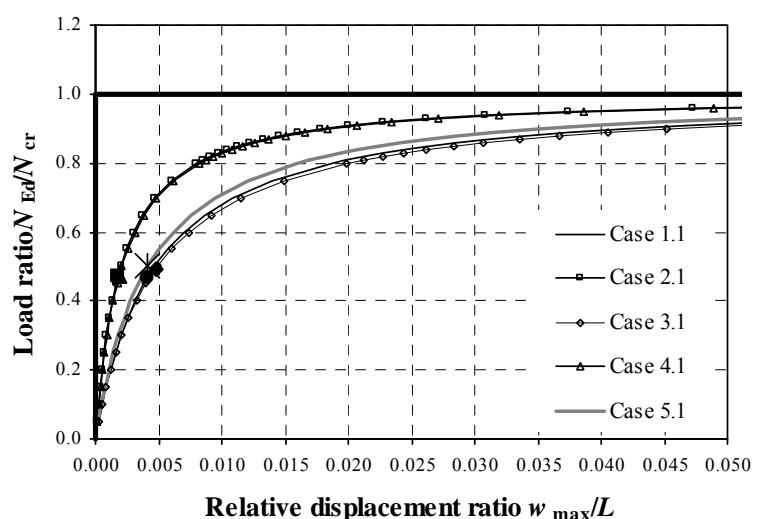

(ii)

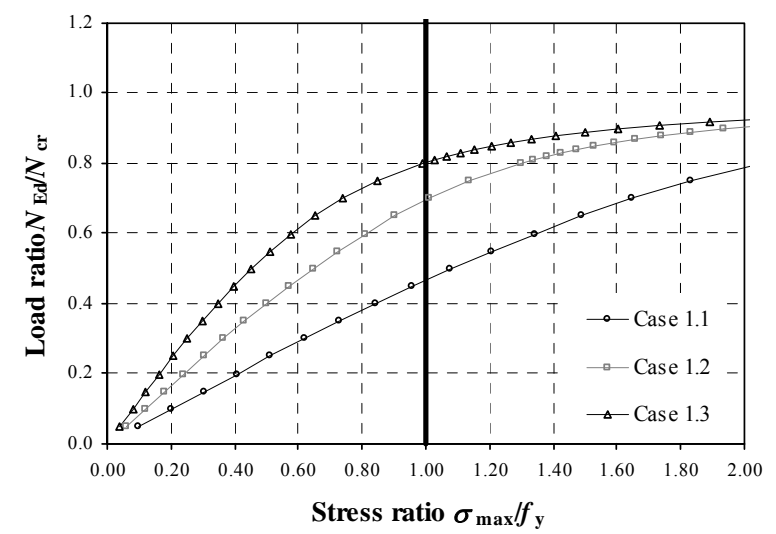

(c)

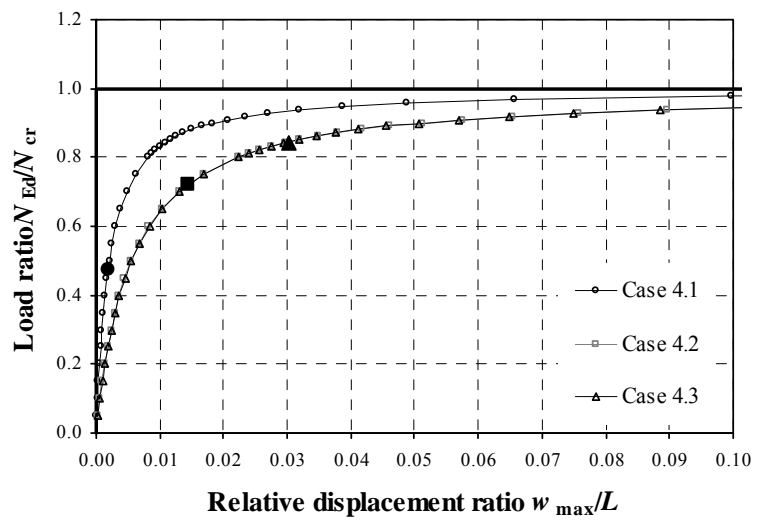

(ii)

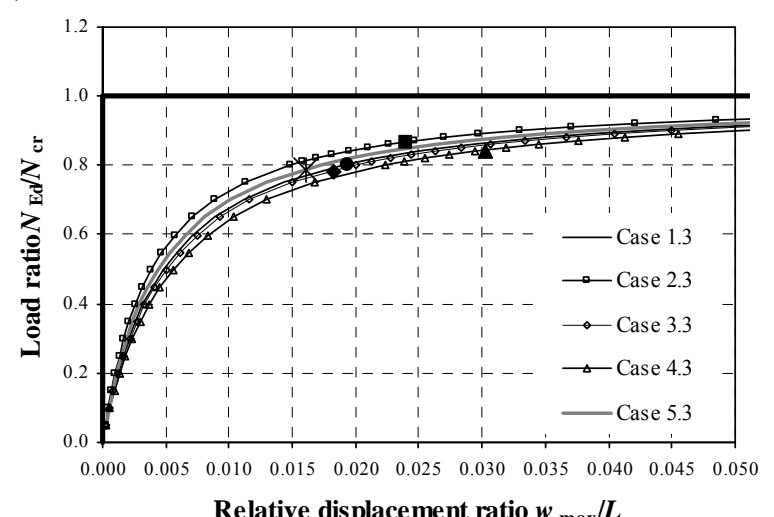

Fig. 20. Example 1. (a) Case 1 - equal rotational end restraints $k_{\theta \mathrm{a}}=k_{\theta \mathrm{b}}=5$ : (i) Load $v s$. relative transverse displacement; (ii) Load $v s$. critical stress. (b) Case $2-k_{\theta \mathrm{a}}=5$ and $k_{\theta \mathrm{b}}=1\left(k_{\theta \mathrm{a}} / k_{\theta \mathrm{b}}=5\right)$ : Load $v s$. relative transverse displacement. (c) Case $4-k_{\theta \mathrm{a}}=1$ and $k_{\theta \mathrm{b}}=5\left(k_{\theta \mathrm{a}} / k_{\theta \mathrm{b}}=0.2\right)$ : Load $v s$. relative transverse displacement. (d) Comparisons for identical ratios $L_{\text {eff }} / L$ (i) $L_{\text {eff }} / L=0.75$; (ii)

$$
L_{\text {eff }} / L=1.25 \text {. }
$$


(a)

(i)

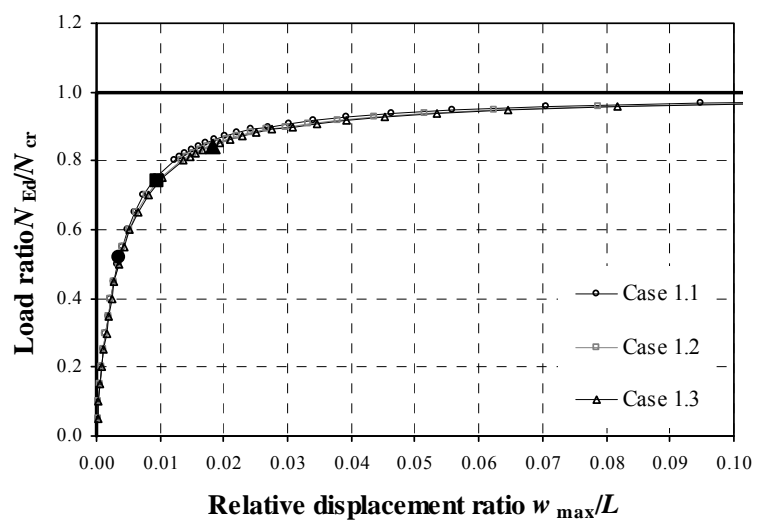

(b)

(i)

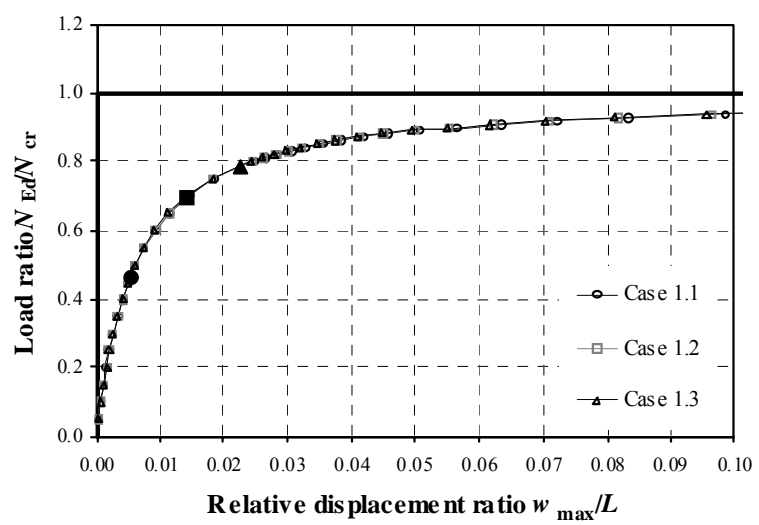

(ii)

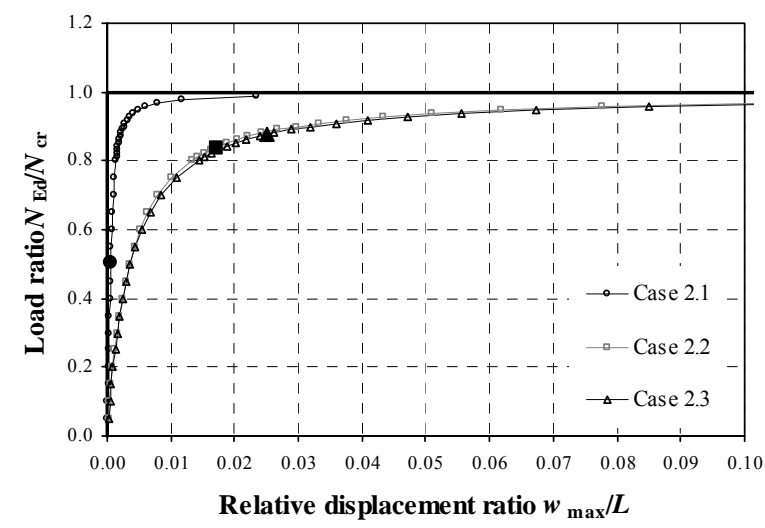

(ii)

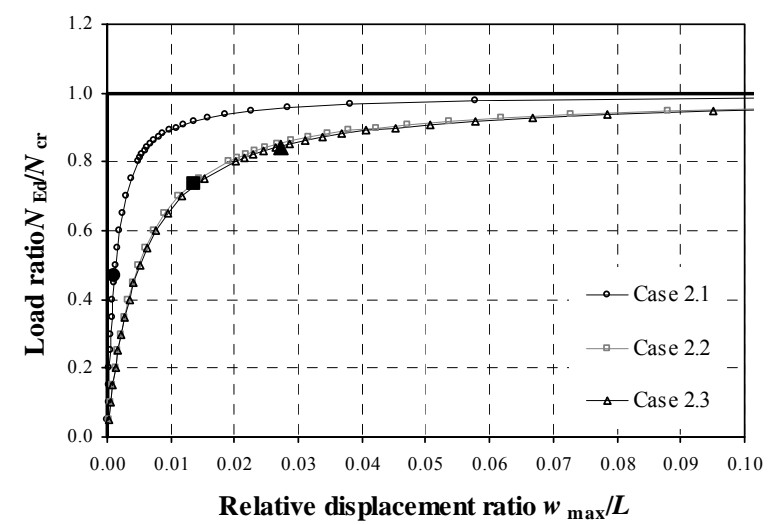

Fig. 21. Equilibrium paths. (a) Example 2: (i) Case 1; (ii) Case 2. (b) Example 3: (i) Case 1; (ii) Case 2. 


\section{List of Tables}

Table 1. Critical loads $N_{\text {cr }}$ and strength ratios (normalized with $N_{\text {cr) }}$ ) for different imperfection levels.

Table 2. Numerical examples and parametric variations in sway imperfection and maximum initial bow amplitude.

Table 3. Strength ratios to the critical load: summary of results.

Table 4. Strength ratios to the critical load: summary of results for different column end restraints.

Table 5. Numerical examples for different soliton parameters.

Table 6. Numerical examples and parametric variations in elastic end-restraints.

Table 7. Strength ratios to the critical load: summary of results.

\section{List of Figures}

Fig. 1. Elastically supported column with equivalent geometric imperfections.

Fig. 2. Free-body diagram of a column segment.

Fig. 3. Form of localized imperfection $w_{0, \text { sol }}$.

Fig. 4. Summary of the basic problem.

Fig. 5. Axially loaded pin-ended column with various amounts of initial bow. (a) Load versus total transverse displacement. (b) Load $v s$. critical stress.

Fig. 6. Equivalent pin-ended columns with initial bow affine to the first mode shape.

Fig. 7. Axially loaded columns with various amounts of initial bow. (a) and (b) Load versus transverse displacement. (c) Load vs. critical stress: columns without sway. (d) Load versus critical stress: columns with sway.

Fig. 8. Combination of initial imperfections with increasing initial bow amplitudes: positive $\Delta_{0}$ and $\delta_{0}$; positive $\Delta_{0}$ and negative $\delta_{0}$. (a) Fourier sine series truncated at $n=1$. (b) Fourier sine series truncated at $n=2$ and $\delta_{1}=0.5 \delta_{2}$ (e.g. cantilevered column).

Fig. 9. Axially loaded sway-prevented (fixed-fixed) column with various amounts of initial bow. (a) Load vs. maximum total transverse displacement for $\Delta_{0}=L / 200$. (b) Comparisons for different sway displacements.

Fig. 10. Axially loaded sway-permitted column with various amounts of initial bow. (a) Cantilevered column (i) Load vs. maximum total transverse displacement for $\Delta_{0}=L / 200$; (ii) Comparisons for different sway displacements. (b) Fixed-guided column (i) Load versus maximum total transverse displacement for $\Delta_{0}=L / 200$; (ii) Comparisons for different sway displacements.

Fig. 11. Initial imperfection shapes represented a Fourier sine series with $n=2$.

Fig. 12. Typical load-deflection curves for pin-ended columns. (a) Load versus total transverse displacement at column mid-height (i) Example $2\left(\delta_{0}=L / 500\right)$; (ii) Example $4\left(\delta_{0}=L / 125\right)$. (b) Comparisons for different sway displacements (examples 4 and $6, \delta_{0}=L / 125, \Delta_{0}=L / 200$ and $\Delta_{0}=$ $L / 500$, respectively).

Fig. 13. Typical load-deflection curves for fixed-guided columns. (a) Load versus total transverse displacement at the column top (i) Example $2\left(\delta_{0}=L / 500\right)$; (ii) Example $4\left(\delta_{0}=L / 125\right)$. (b) Comparisons for different sway displacements (examples 4 and $6, \delta_{0}=L / 125, \Delta_{0}=L / 200$ and $\Delta_{0}=$ $L / 500$, respectively). 
Fig. 14. Axially loaded sway-prevented columns. (a) Pin-ended column (i) $\delta_{0}=L / 500$; (ii) $\delta_{0}=$ $L / 125$. (b) Fixed-fixed column (i) $\delta_{0}=L / 500$; (ii) $\delta_{0}=L / 125$.

Fig. 15. Axially loaded sway-permitted columns. (a) Cantilevered column (i) $\delta_{0}= \pm L / 500$; (ii) $\delta_{0}=$ $\pm L / 125$. (b) Fixed-guided column (i) $\delta_{0}=L / 500$; (ii) $\delta_{0}=L / 125$.

Fig. 16. Typical load-deflection curves for pin-ended columns with local imperfections modelled as solitons. (a) Effect of the wave amplitude and location. (b) Effect of form parameter $\varphi_{\text {sol }}$.

Fig. 17. Typical load-deflection curves for pin-ended columns with combined localized and single half-sine imperfections. (a) Effect of the location of the soliton peak (i) $0.25 \mathrm{~L}$; (ii) $0.50 \mathrm{~L}$. (b) Effect of initial bow amplitude and soliton orientation. (c) Effect of the soliton wave amplitude and orientation.

Fig. 18. Critical spring stiffness model. (a) Pinned ends. (b) Rigid ends. (c) Elastically restrained ends.

Fig. 19. Typical curves demonstrating the transition from sway-permitted columns to partly sway and nearly fully sway-prevented. (a) $k_{\theta \mathrm{a}}=k_{\theta \mathrm{b}}$. (b) $k_{\theta \mathrm{a}}=0$. (c) $k_{\theta \mathrm{a}}=\infty$.

Fig. 20. Example 1. (a) Case 1 - equal rotational end restraints $k_{\theta \mathrm{a}}=k_{\theta \mathrm{b}}=5$ : (i) Load $v s$. relative transverse displacement; (ii) Load versus critical stress. (b) Case $2-k_{\theta \mathrm{a}}=5$ and $k_{\theta \mathrm{b}}=1\left(k_{\theta \mathrm{a}} / k_{\theta \mathrm{b}}=5\right)$ : Load versus relative transverse displacement. (c) Case $4-k_{\theta \mathrm{a}}=1$ and $k_{\theta \mathrm{b}}=5\left(k_{\theta \mathrm{a}} / k_{\theta \mathrm{b}}=0.2\right)$ : Load versus. relative transverse displacement. (d) Comparisons for identical ratios $L_{\text {eff }} / L$ (i) $L_{\text {eff }} / L=0.75$; (ii) $L_{\text {eff }} / L=1.25$.

Fig. 21. Equilibrium paths. (a) Example 2: (i) Case 1; (ii) Case 2. (b) Example 3: (i) Case 1; (ii) Case 2. 